\title{
Optical coherence tomography imaging in uveitis
}

\author{
Sumru Onal • Ilknur Tugal-Tutkun • \\ Piergiorgio Neri · Carl P Herbort
}

Received: 26 May 2013/Accepted: 24 June 2013/Published online: 9 July 2013

(C) Springer Science+Business Media Dordrecht 2013

\begin{abstract}
Optical coherence tomography (OCT) is a non-contact noninvasive technique that allows in vivo imaging of the retina, choroid, optic nerve head, retinal nerve fiber layer, and the anterior structures of the eye. It was introduced into clinical practice two decades ago. Advances in OCT technology have been achieved by searching ultra-high-resolution OCT, adaptive optics OCT, eye-tracking OCT, and changes in signal detection technique from time-domain (TD) to spectral-domain (SD) detection. Today, SD OCT
\end{abstract}

S. Onal $(\square)$

Department of Ophthalmology, Koc University School of Medicine, Istanbul, Turkey

e-mail: sumruo_md@yahoo.com

S. Onal

Department of Ophthalmology, V.K. Foundation American Hospital, Istanbul, Turkey

I. Tugal-Tutkun

Department of Ophthalmology, Istanbul Faculty of Medicine, Istanbul University, Istanbul, Turkey

P. Neri

The Eye Clinic, Polytechnic University of Marche, Ancona, Italy

C. P Herbort

University of Lausanne, Lausanne, Switzerland

C. P Herbort

Retinal and Inflammatory Eye Diseases, Centre for Ophthalmic Specialised Care (COS), Lausanne,

Switzerland has become a part of routine uveitis practice. Apart from its diagnostic value in uveitis, OCT has enabled objective assessment of treatment response and provided predictive value for visual recovery and prognosis of uveitic entities. It is the standard diagnostic technique in the detection, monitoring of treatment, and determination of prognosis in uveitic macular edema as well as other inflammatory macular pathologies, including epiretinal membrane formation, vitreomacular traction, foveal atrophy, and lamellar/full-thickness macular holes. OCT has also shed light on the pathophysiology of several posterior uveitic entities. SD OCT has enabled visualization of four lines in the sensory retina which represent the external limiting membrane, the photoreceptor inner and outer segment junction, the photoreceptor outer segment and the retina pigment epithelium junction, and the retina pigment epithelium-choriocapillaris complex. Thus, we have gained substantial information about the pathologic and structural changes in uveitic conditions with primary or secondary outer retinal involvement. SD OCT has also provided invaluable information on the inner retinal and the vitreoretinal interface changes in uveitic conditions. With the introduction of enhanced depth imaging, visualization of the choroid and choriocapillaries has become possible. Therefore, OCT has become an indispensible ancillary test in the diagnosis and management of inflammatory diseases involving the retina and/or the choroid. As OCT technology continues to develop further it will provide new insights 
into the retinal and choroidal structure and the pathogenesis of posterior uveitic entities.

Keywords Optical coherence tomography - Timedomain · Spectral-domain · Enhanced depth imaging . Uveitis

\section{Introduction}

Definition

Optical coherence tomography (OCT) is a rapid noncontact method that allows in vivo imaging of the retina, choroid, optic nerve head, retinal nerve fiber layer (RNFL), and the anterior segment structures of the eye. It provides high-resolution cross-sectional images of tissue morphology both in situ and in real-time. The ability of OCT to image tissue morphology in situ and in real-time has been termed 'optical biopsy'.

\section{Historical background}

OCT was introduced in 1991 as a noninvasive in vivo imaging technique [1]. In vivo imaging of the healthy retina and optic nerve head and of retinal diseases as well as the ease of obtaining these images changed the diagnostic strategy used by ophthalmologists [2-6]. OCT was introduced commercially in 1996. Early models (OCT 1 and OCT 2; Carl Zeiss Meditec, Dublin, CA, USA) produced axial resolution at $12-15 \mu \mathrm{m}$. In 2002, a third generation time-domain (TD) OCT 3 (Stratus OCT; Carl Zeiss Meditec) became available with an axial resolution of $10 \mu \mathrm{m}$ and a scan velocity of 400 axial scans/sec. Since 2004, higher resolution spectral-domain OCT (SD OCT) has entered clinical practice with reported resolutions of $3-5 \mu \mathrm{m}$ as well as improved visualization of retinal morphologic and pathologic features [7-14]. SD OCT is the current gold standard for posterior segment retinal tomography.

\section{Technique}

OCT imaging is analogous to ultrasound; however, it uses light instead of sound to acquire high-resolution images of ocular structures. Measurements are obtained by directing a beam of light onto tissue and measuring the echo time delay and magnitude of reflected or backscattered light using low-coherence interferometry.

Time-domain optical coherence tomography

In TD OCT technology low-coherence, near-infrared light from a superluminescent diode light source is split to go to a reference mirror and to the retina. As the light comes back from the mirror and the eye, it creates an interference pattern, which is then analyzed by a photodetector and an image is created. In this technology, an individual A-scan is acquired by varying the length of the reference arm in an interferometer, such that the scanned length of the reference arm corresponds to the A-scan length. Cross-sectional images are generated by scanning the optical beam in the transverse direction, thereby yielding a two-dimensional data set (B-scan) that can be displayed as a false color or gray-scale image [1]. With the commercially available machine, Stratus OCT, axial resolutions of $10 \mu \mathrm{m}$ can be achieved, and cross-sectional retinal images consisting of $512 \mathrm{~A}$-scans can be acquired in $1.28 \mathrm{~s}$. For scanning of the optic nerve head or the macula with TD OCT, six radial line scans in a spokelike pattern are centered on either the optic nerve head or the fovea, respectively. OCT interpolates to fill in the gaps of information between the radial line scans.

Spectral-domain optical coherence tomography

Until 2006, all commercially available OCT machines were based on TD OCT, in which depth information is acquired sequentially with a moving reference mirror and by looking at the change of interference pattern in time. Advances in OCT technology have been achieved by exploring ultra-high-resolution (UHR) OCT, adaptive optics OCT, eye-tracking OCT, and changes in signal detection technique from TD to SD detection [15]. SD OCT uses essentially the same system as the conventional TD OCT. It may use either a superluminescent diode or femtosecond titaniumsapphire laser as a light source. In contrast to TD OCT, the reference mirror for SD OCT remains stationary, and the depth information is acquired by analyzing the interference patterns in a spectrum of mixed reflected lights [7-14]. SD OCT technology uses low-coherence 
interferometry to detect light echoes, relying on a spectrometer and high-speed camera and based on the mathematical premise of Fourier transformation. Because application of Fourier mathematical formula has the effect of measuring all echoes of light simultaneously, as compared with sequentially in the case of TD OCT, SD OCT significantly increases the amount of data acquired in each session, resulting in a significant reduction of motion artifacts and an increased signal-to-noise ratio compared to TD OCT. Because the SD technique shows a higher sensitivity (i.e., signal-to-noise ratio) than the TD technique, the cross-sectional images obtained by the currently available SD OCT instruments have both a higher axial resolution and sensitivity than those obtained by Stratus OCT. Less interpolation between scans is required, making volumetric analysis and 3-dimensional (3D) imaging possible. Increased amounts of data allow image registration, which aids in improved visit-to-visit measurement reproducibility. Commercially available SD OCT systems possess image acquisition speeds between 18,000 and 53,000 A-scans/sec [16].

To overcome motion artifacts caused by fixational eye movements in OCT imaging, several commercial systems have implemented eye tracking into their existing OCT devices e.g., Spectralis HRA and SD OCT device (Heidelberg Engineering, Heidelberg, Germany), RTVue (Optovue Inc., Fremont, CA, USA) and Cirrus HD-OCT (Carl Zeiss Meditec). The Spectralis and RTVue systems use image-based realtime eye tracking in which eye motions are extracted from video frames and the OCT beam is steered to keep it on target. The built-in real-time eye movement tracking system recognizes features in the scanning laser ophthalmoscope (SLO) image including blood vessels and the optic disc. This enables stabilization of the scan coordinates in relation to the retina coordinates and minimizes motion artifacts during image acquisition. In the Spectralis system, the retinal image (1,000 points) from the infra-red SLO imaging channel is used to track motion. In the RTVue system the motion is measured at $30 \mathrm{~Hz}$ from an infrared fullfield fundus camera [17, 18]. The Cirrus system reduces eye motion artifacts with a proprietary scan acquisition strategy, a high-speed $20 \mathrm{~Hz}$ line scanning ophthalmoscope (LSO) camera, and single-pass alignment scanning. The retinal tracking system locks onto the patient's retina to negate eye movement and enables tracking of the current scan to the position of a prior scan. During acquisition, proprietary algorithms automatically detect motion and track the eye.

The currently available SD OCT instruments allow improved visualization of four lines in the sensory retina, which represent the external limiting membrane (ELM), the photoreceptor inner and outer segment (IS/OS) junction, the interdigitation of the photoreceptor OS and the retina pigment epithelium (RPE) or OS/RPE junction, and the RPE-choriocapillaris complex. The OS/RPE junction line is also named cone outer segment tip (COST) line and represents a thin line between the IS/OS junction and RPE [19]. However, lately Spaide and Curcio have raised a debate regarding the anatomical assignments of these four lines. By creating a scale model of outer retinal morphology from published information and by comparing this information with SD OCT scans the authors argued that the four outer lines seen are the ELM, the ellipsoid section of the photoreceptors, the contact cylinder of the cones, and finally the RPE. Studies comparing OCT in vivo with histologic correlative evaluations are currently underway and may lead to definitive assignments of these outer lines [20]. Nevertheless, all four lines are diagnostically useful in understanding the pathologies and structural changes of the retina. Figure 1 shows a high-resolution SD OCT scan of the normal retina.

Furthermore, several of the SD OCT devices incorporate overlay software that permits correlation of OCT images with photographic, angiographic, autofluorescent, and other studies. Nine different posterior segment SD OCT systems are currently commercially available. There are also reports from separate groups who have designed customized SD OCT devices and software [21-23].

Ultra-high-resolution optical coherence tomography

On a commercial basis, further improvement of B-scan images has been achieved by using UHR SD OCT and speckle noise-reduction technology with SD OCT. UHR SD OCT using a femtosecond titaniumsapphire laser light source achieves a $3 \mu \mathrm{m}$ B-scan image with a high contrast and allows highly detailed imaging of pathologies. However, the expensive cost of the titanium-sapphire laser light source hinders wide commercial use of this technique. Using a light- 


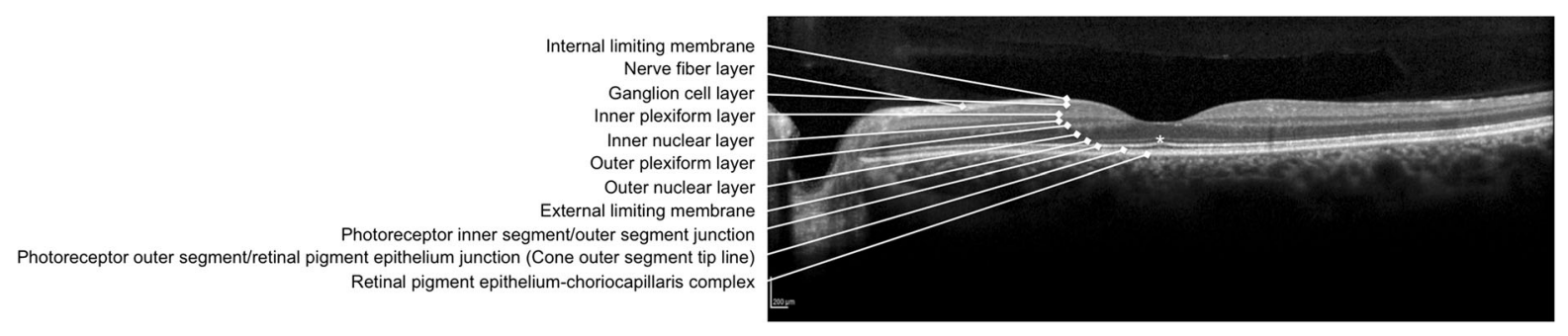

Fig. 1 High-resolution SD OCT shows normal macular architecture on a cross-sectional B-scan. Asterisk represents the normal foveal bulge. SD OCT image of a 9-mm scan was

source system composed of two superluminescent diode lasers with different central wavelengths has also been shown to be effective in achieving $3 \mu \mathrm{m}$ B-scan images. The SOCT Copernicus HR (Canon/ Optopol, Inc.), a commercial prototype of the UHR imaging procedure, employs two superluminescent diode light-source systems and has achieved an axial resolution of $3 \mu \mathrm{m}$.

Another approach to improve B-scan images is to reduce speckle noise via multiple B-scan averaging ('enhanced SD OCT') [15]. In the context of OCT, a speckle is defined as a $3 \mathrm{D}$ volume in the tissue, from which the returning light causes interference with the light from the reference arm. In OCT B-scan images, the dominating noise that obscures the fine retinal structures is usually the speckle noise arising from the interference of coherent waves backscattered from nearby scatterers [24]. The reduction of speckle noise in SD OCT enables highly detailed imaging of pathologies in the photoreceptor and RPE layer [15]. The Spectralis HRA and SD OCT device (Heidelberg Engineering), which shows an axial resolution of only $7 \mu \mathrm{m}$, has enabled the most effective reduction of speckle noise by more precise multiple B-scan averaging.

\section{Enhanced depth imaging}

Recently, a technique termed 'enhanced depth imaging' (EDI) has been described by Margolis and Spaide, which uses the commercially available Spectralis HRA and SD OCT device (Heidelberg Engineering) to obtain an enhanced view of the choroid [25]. By placing the OCT machine close enough to the eye to acquire an inverted image within a $5^{\circ}-30^{\circ}$ area centered at the fovea and then performing manual measurements from the outer border of RPE to the inner sclera border the OCT scan

obtained using Heidelberg Spectralis OCT with a custom scan acquisition protocol of up to 25 raster lines of $9 \mathrm{~mm}$ scan length with automatic real-time imaging set at 30 images

is placed on the choroid. This has been successfully used to provide in vivo, cross-sectional, histologic information of the choroid, such as choroidal thickness measurements, and imaging of choroidal abnormalities, such as central serous chorioretinopathy, choroidal neovascularization, and choroidal rupture. A pilot EDI SD OCT study with normal eyes of Caucasians has shown choroidal thickness of the right and left eye to be 318 and $335 \mu \mathrm{m}$, respectively [26]. Because the choroid is a frequent target for intraocular inflammation, in vivo imaging of the choroidal vasculature can be helpful in the evaluation of specific disease processes. Figure 2 shows EDI OCT of a normal subject.

\section{En-face optical coherence tomography}

Podoleanu et al. [24-30] developed a system combining confocal ophthalmoscopy and OCT, producing en-face OCT images that can be directly compared with transversal images provided by the confocal channel. In OCT, the depth resolution is determined by the bandwidth of the optical source used; therefore, en-face OCT and conventional longitudinal OCT achieve the same depth resolution as long as the source used is the same. In conventional longitudinal OCT images, the longitudinal B-scans are built up from several parallel A-scans along the $\mathrm{Z}$ axis (i.e., depth) in the $\mathrm{X} / \mathrm{Z}$ or $\mathrm{Y} / \mathrm{Z}$ plane. En-face OCT enables the production of transversal $\mathrm{C}$-scans in the $\mathrm{X} / \mathrm{Y}$ plane at a fixed $\mathrm{Z}$ coordinate. By changing the $\mathrm{Z}$ coordinate in the OCT channel, OCT C-scans are taken at different depths in the retina [31].

Further advances

Several technologies are currently under development. Already integrated in some platforms, Doppler OCT 


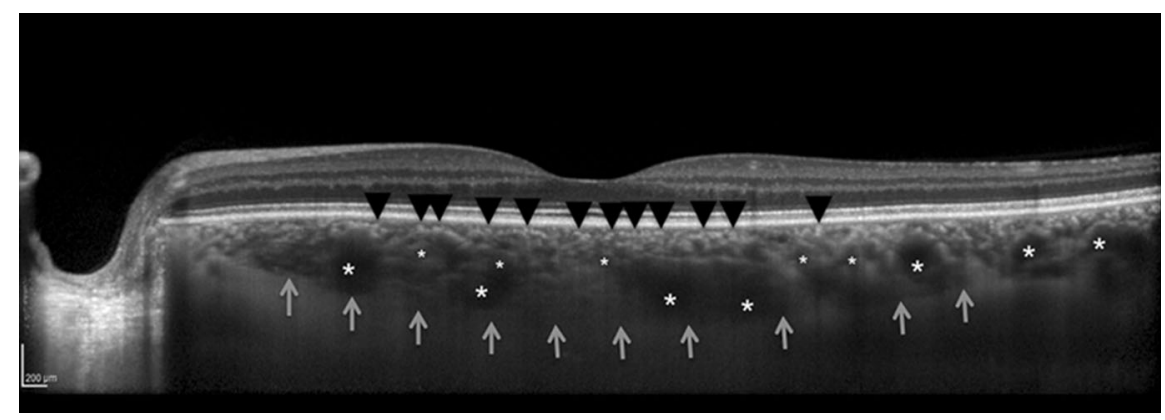

Fig. 2 EDI OCT of a normal subject shows the whole thickness of the choroid from the Bruch membrane to the suprachoroidal space (gray arrows). Black arrowheads exemplify hyperreflective dots in the inner choroid that represent cross-sectional views of parallel-lying pericapillary arterioles and venules. Asterisks

can measure blood flow in the retinal and choroidal vessels [32]. Swept-source OCT can achieve ultrahigh axial resolution by sweeping a narrow bandwidth light source through a broad optical range [33]. Scattering optical coherence angiography will be able to visualize the choroidal vasculature of the macula and the optic nerve head without intravenous dye injection [34]. We believe that such technology could be extensively useful in understanding the vascular pathology involving the retina and choroid in eyes with posterior uveitis.

High-penetration posterior OCT (HP OCT) systems, which use a longer wavelength for the probe than standard penetration OCT (1060 vs. $830 \mathrm{~nm})$, were demonstrated to have higher penetration into the choroid [35-37]. The longer wavelength is less affected by the scattering of the sample and the absorption of retinal pigments and thus enables high-contrast imaging of the choroid. This technology is also useful to assess uveitis entities with primary choroidal involvement as well as to document the presence of complications such as choroidal neovascular membranes.

\section{Limitations}

Because OCT utilizes light instead of sound, media opacities can interfere with imaging. A clear media is required for optimal imaging. Eyes with significant corneal opacities, dense cataract, and significant vitreous haze or hemorrhage are not amenable to OCT imaging. However, SD OCT has been shown to be superior to TD OCT in uveitic eyes by providing represent medium- and large-sized choroidal vessels. EDI OCT of a $9 \mathrm{~mm}$ line scan centered at the fovea was obtained using Heidelberg Spectralis OCT with automatic real-time image averaging set at 100 images

better identification of normal and pathologic macular structures in patients with poor media clarity [38]. As with most diagnostic tests, patient cooperation is necessary for optimal imaging in OCT and patient movement can diminish the image. With SD OCT machines, a shorter acquisition time and fixationindependent scan adjustment resulted in fewer motion-related artifacts. Early models of OCT relied on the operator to accurately place the image over the desired pathology. Point-to-point registration with eye tracking featured with SD OCT technology has overcome acquisition errors and enabled a high intraobserver reproducibility allowing determination of minimal changes that may occur between successive examinations. An established limitation of SD OCT is shadowing of the underlying retinal structures by hyperreflective lesions such as exudates, hemorrhages and major retinal vessels leading to loss of details of the underlying morphology.

\section{Use of optical coherence tomography technology in uveitis practice}

Imaging anterior chamber inflammatory reaction in uveitis

Slit-lamp biomicroscopy is the standard method for assessment of the inflammatory reaction in the anterior chamber. An adaptation of OCT technology for better visualization of the anterior segment has been achieved through using a longer wavelength light of $1,310 \mathrm{~nm}$ compared with $830 \mathrm{~nm}$ for retinal imaging. 
In one study, cross-sectional images of the anterior chamber were obtained using anterior segment OCT (AS OCT) (Visante; Carl Zeiss Meditec) and hyperreflective spots were counted in non-enhanced images manually and with automated computer algorithms [39]. There was good correlation between automated and manual methods in grading the hyperreflective spots. The automated method was more sensitive in detecting hyperreflective dots in eyes with $4(+)$ cells in the anterior chamber. Aqueous flare was not detected in eyes except with 4(+) flare and fibrinous reaction. Notably, AS OCT was able to detect anterior chamber cells in eyes with significant corneal edema ranging from $702-1,020 \mu \mathrm{m}$. The ability of AS OCT to detect anterior chamber cells in eyes with corneal haze or edema is the most striking advantage of this new modality.

The AS of one patient with sclerokeratouveitis presumed to be of tuberculous etiology was evaluated using AS OCT [40]. AS OCT showed a poorly demarcated amorphous lesion in the iridocorneal angle, corneal edema, narrowing and synechiae of the iridocorneal angle, and anterior chamber exudates and cells. Improvement of corneal edema and a decrease of corneal thickness and exudates were observed after anti-tuberculosis treatment was begun. AS OCT was shown to be useful in assessing the extent of anterior synechiae and angle lesions. It allowed noninvasive non-contact analysis of treatment response and was useful to assess disease activity.

Because the pigmented posterior layer of the iris prevents light transmission beyond this structure, the ciliary body cannot be visualized using AS OCT. Therefore, the current technology is not applicable for evaluation of the ciliary body and the pars plana in intermediate uveitis, and complications arising at this anatomical space.

Uveitic macular edema and other macular pathological findings

Today, OCT has become a standard diagnostic technique for the evaluation of uveitic macular edema and other pathologies involving the macula in patients with uveitis. The currently increased resolution and improved image quality along with the ease of obtaining these images have augmented its significance for macular diagnostics in uveitis practice. Gupta et al.
[38] compared the performance of TD OCT (Stratus OCT) and SD OCT (Cirrus HD-OCT) in patients with uveitis with vitreous haze $1(+)$ or worse. Preliminary results suggested that SD Cirrus HD-OCT has an advantage over TD Stratus OCT in uveitic eyes by providing better identification of normal and pathologic structures in patients with poor media clarity. Compared with TD Stratus OCT, SD Cirrus HD-OCT was able to detect internal limiting membrane (ILM) folds, posterior vitreous detachment, cystoid retinal spaces, outer lamellar hole, and disruption of the IS/OS junction in eyes where conventional TD OCT could not. Additionally, Cirrus HD-OCT was able to diagnose vitreomacular traction in eyes that produced normal results on Stratus OCT. Roesel et al. [41] evaluated uveitic macular edema using TD OCT (Stratus OCT) and SD OCT (Spectralis OCT) and confirmed the advantages of SD OCT for defining macular morphologic features in uveitis patients with macular edema, including detection of the IS/OS junction. The authors also concluded that loss of the IS/OS junction on OCT in eyes with uveitic macular edema was associated with poor visual acuity. Foveal thickness measured by SD was higher than that measured with the TD OCT. Several reasons have been shown to account for this variability. First, SD OCT measures the retinal thickness from RPE to the ILM, whereas TD Stratus OCT measures the retinal thickness from IS/OS to the ILM, excluding a significant part of the structure for retinal thickness measurements. The distance between IS/OS and RPE is approximately $45 \mu \mathrm{m}$; thus, SD OCT would be inherently thicker compared to TD OCT. Second, the retinal thickness measurement in Stratus OCT may be biased by the retinal thickness in the central subfield alone as only six linear line scans pass through the foveal center. SD OCT, on the other hand, measures the retinal thickness by an evenly distributed square centered on the fovea and is likely to indicate the true retinal thickness. Another established important feature of SD OCT is a high intraobserver reproducibility of macular thickness measurements in eyes with uveitic macular edema allowing the determination of a minimal thickness change to occur as a consequence of treatment [42].

Optical coherence tomography is suitable for detecting and monitoring uveitic macular edema [43, 44]. It provides important information about the fluid distribution in eyes with macular edema and the morphology of the vitreoretinal interface. Markomichelakis et al. [45] using OCT 1, first described three 
different patterns of distribution of fluid in the macula of patients with uveitis-cystoid macular edema (CME), diffuse macular edema (DME), and serous retinal detachment. A similar classification of morphological features on OCT exists in patients with diabetic macular edema and has been correlated with focal and diffuse fluorescein leakage patterns by Byeon et al. [46]. CME signifies the presence of low-reflective intraretinal spaces separated by thin, high-reflective retinal tissue (Fig. 3). DME is characterized by increased macular thickness and the presence of small low-reflective areas with spongy appearance of the retinal layers. Serous retinal detachment is seen as a clear separation of the neurosensory retina from the RPE (Fig. 3). The relative frequency of the three different patterns in uveitis varies depending on the patient selection criteria [45, 47, 48]. Both CME and DME can potentially be found with serous retinal detachment. Other authors using OCT 3 then confirmed the initial classification [48-50]. Another important feature confirmed by the studies was the presence of epiretinal membrane (ERM). On OCT, an ERM appears as a hyperreflective line adhering to the retina (Fig. 4). OCT showed ERM in a higher percentage of cases compared to those observed ophthalmoscopically in patients with uveitis. In a significant amount of eyes with ERM detected ophthalmoscopically, OCT also revealed concurrent vitreoretinal traction (Fig. 5). These data confirm the high sensitivity of OCT in showing the presence of ERM and potential vitreoretinal abnormalities in the macular area, suggesting the hypothesis of a tractional mechanism as a probable origin or cofactor of the onset of macular edema during uveitis. The presence of an ERM has been found to be independent from the site of inflammation, from different types of edema, and from the macular thickness [49].

Tran et al. [50], using Stratus OCT, tried to associate OCT patterns with particular fluorescein angiography (FA) findings in uveitis patients with macular edema. They also investigated the correlation between tomographic features and visual acuity and determined the impact of OCT patterns on visual improvement over time. In $86.8 \%$ of the eyes with uveitic macular edema, similar results were detected on both OCT and FA. In $6.2 \%$ of eyes serous retinal detachment was detected on OCT, but there was no perifoveal hyperfluorescence on FA, and in $7 \%$ of eyes there was perifoveal hyperfluorescence on FA but
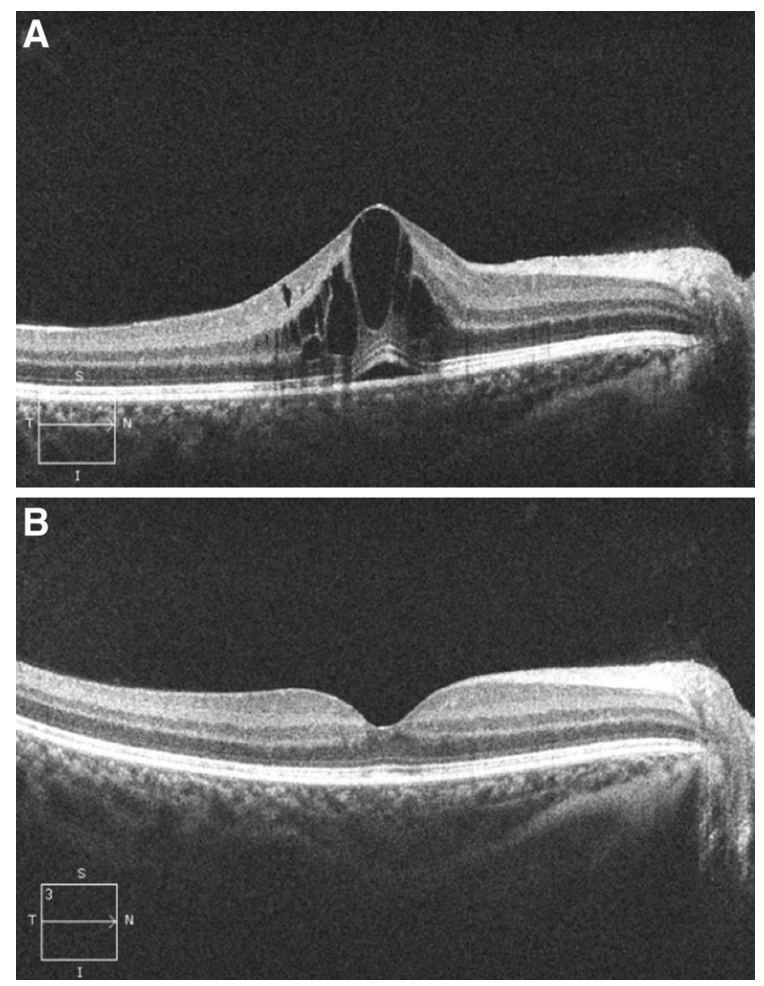

Fig. 3 Cystoid macular edema and serous retinal detachment on a cross-sectional macular B-scan (a). Resolution of cystoid spaces and subfoveal fluid following treatment (b). SD OCT image of $9 \mathrm{~mm}$ scans were obtained using Cirrus HD-OCT with high-definition 5-line scan mode

no intraretinal cysts on OCT. Visual acuity decreased with increasing fluorescein leakage and with increasing central thickness. A correlation was found between central thickness measured by OCT and visual acuity. This correlation showed significant difference depending on the OCT pattern and was strongly dependent on the presence of CME. A negative correlation between central foveal thickness and visual acuity has also been described in other studies [43, 45, 47, 49]. Although DME was found to be associated with poor visual recovery after treatment [50], some studies point to a negative visual outcome in patients with uveitic CME [45, 48, 51, 52]. The presence of serous retinal detachment in eyes with uveitic macular edema has been shown not to worsen the visual prognosis [50, 53]. Lehpamer et al. [54], using SD OCT, analyzed the effect of presence of subretinal fluid in patients with uveitic macular edema on visual acuity and response to therapy. The authors concluded that presence of subretinal fluid on OCT was associated with greater 

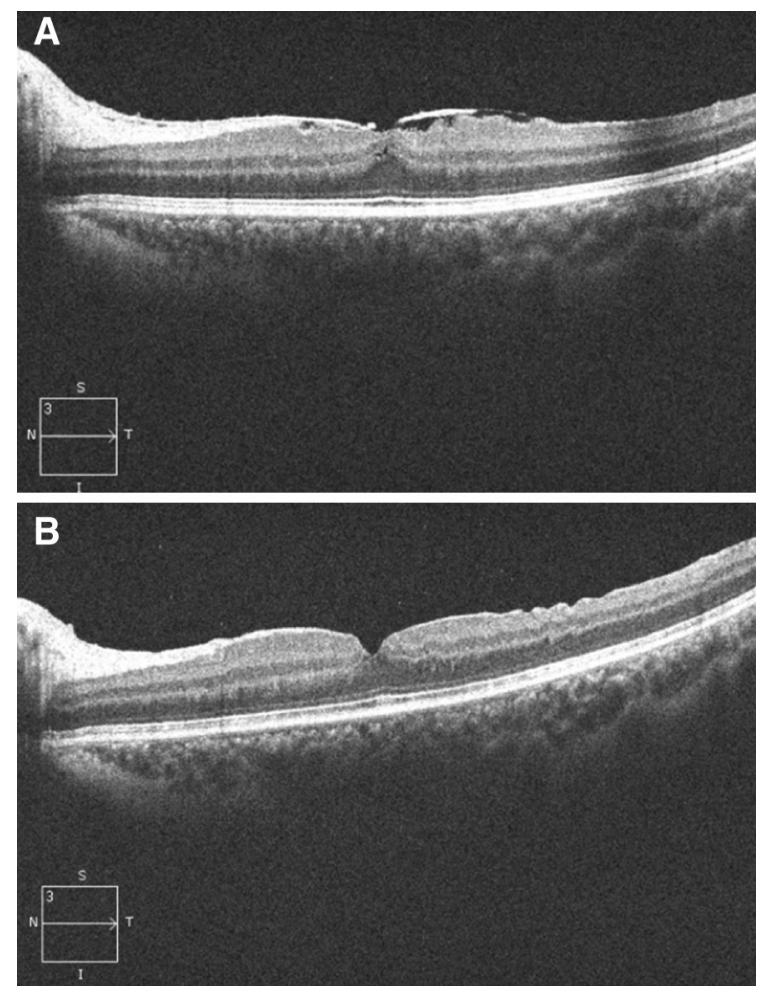

Fig. 4 HD OCT shows a segmented hyperreflective line adhering to the inner retina representing an epiretinal membrane (ERM) on a cross-sectional macular B-scan; the ERM leads to formation of inner retinal wrinkling (a). OCT shows persistent inner retinal wrinkling two months after pars plana vitrectomy, membrane peeling, and gas-fluid exchange (b). SD OCT image of $9 \mathrm{~mm}$ scans were obtained using Cirrus HD-OCT with highdefinition 5-line scan mode

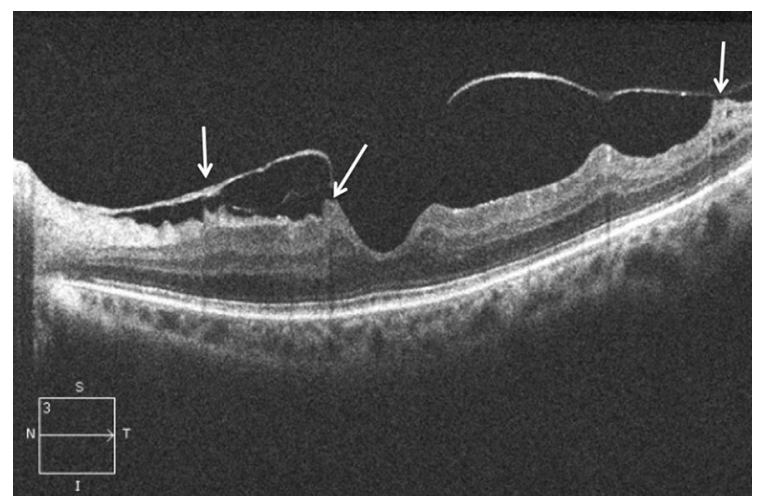

Fig. 5 High-definition SD OCT shows vitreoretinal traction on a cross-sectional B-scan; arrows indicate areas of vitreomacular traction. SD OCT image of $9 \mathrm{~mm}$ scans was obtained using Cirrus HD-OCT with high-definition 5-line scan mode macular thickness and worse visual acuity at presentation. However, eyes with serous retinal detachment and macular edema responded favorably to uveitis treatment at 3 and 6 months, achieved greater rates of improvement and resolution of macular edema than eyes without serous retina detachment, and recovered to a similar level of visual acuity.

An unusual finding described in eyes with active iridocyclitis using Stratus OCT is the occurrence of a ring-like pattern of retinal thickening outside the foveal center in the inner ring and combined inner and outer rings [55-57]. The macular thickening has been shown to have a significant and consistent correlation with active iridocyclitis [55]. The mechanism underlying the development of non-CME in eyes with iridocyclitis is unknown but is possibly caused by the same pathophysiological mechanisms underlying the development of CME in eyes with anterior uveitis. Although it has been suggested that the ring-like thickening outside the foveal center can persist for 6 months after an acute episode in $45 \%$ of acute anterior uveitis cases [55], one study has shown that the OCT parameters including total macular volume return to normal by 6 months after the uveitis flare-up [58].

Macular edema and/or maculopathy in patients with juvenile idiopathic arthritis (JIA)-associated uveitis has also been studied by OCT [57, 59, 60]. Maculopathy has been documented by OCT in $84 \%$ of eyes with JIA-associated uveitis [57], a much higher figure compared to previous reports mostly based on ophthalmoscopic findings (3.9-26\%) [61-64]. Macular changes determined by OCT included perifoveolar thickening in $74 \%$, macular edema in $48 \%$, foveal detachment in $18 \%$, and atrophic changes in $10 \%$ of eyes with JIA-associated uveitis [57].

Akduman [65] studied the correlation of changes in macular thickness between visits and compared the change to visual acuity, cell, flare, or the inflammation index (which was defined as cell and flare). The best correlations were found between macular thickness and visual acuity, and between the inflammation index and macular thickness. Based on these findings the author concluded that the macular thickness as seen on OCT might be more objective and correlate more closely with visual acuity than measurement of flare and/or cell in eyes with uveitis. Roesel et al. [66] focused on the correlation of fundus autofluorescence (FAF) and OCT with visual acuity in eyes with uveitic macular edema. In 
eyes with macular edema an increased FAF, which probably arises from proteins such as retinoids in the extracellular fluid, has been observed. Using Spectralis OCT, the authors concluded that FAF and OCT are useful diagnostic tools for the evaluation of uveitic macular edema. Increased central FAF, presence of cystoid changes, a disrupted IS/OS junction on OCT, and ERM were associated with poor vision. Roesel et al. [67] also analyzed the correlation of microperimetry (photoreceptor function) and SD OCT with visual acuity in eyes with uveitic macular edema. Foveal thickness and fundus-related microperimetry were correlated with visual acuity. Cystoid changes in the outer plexiform and inner nuclear layer (INL) and the presence of ERM were associated with poor visual acuity. Fixation abnormalities were not associated with poor visual acuity. Georgiadou et al. [68] assessed the correlation of visual acuity, OCT, and multifocal electroretinography (mfERG) in patients with uveitic macular edema before and after treatment. In CME, the value of $\mathrm{mfERG}$ before treatment was related to the central foveal thickness and visual acuity. In contrast, after treatment, the decrease of macular thickness was not always followed by an improvement of mfERG and visual acuity.

Optical coherence tomography has also been used to diagnose, assess, and monitor the therapeutic response in uveitic macular edema [48, 51, 69-85]. In a recent study, Sugar et al. [86] tried to identify a clinically meaningful threshold for change in uveitic macular edema evaluated by OCT. A $20 \%$ change in retinal thickness in patients with uveitic macular edema was found to be optimal for clinically important changes in visual acuity. A threshold for change in retinal center subfield thickness of $20 \%$ balanced the percentage of false positives and false negatives for predicting more than a 10-letter change in visual acuity with a sensitivity of $77 \%$ and a specificity of $75 \%$. For studies of treatments for uveitic macular edema in which improvement is monitored through changes in retinal thickness, this threshold was suggested to be optimal in terms of balancing the sensitivity and specificity associated with clinically meaningful changes in visual acuity. The authors also proposed that this threshold could be considered as an outcome for clinical trials of treatments for uveitic macular edema. Payne et al. [87] determined the utility of logarithmic transformation of SD OCT ( $\log \mathrm{SD}$ OCT) retinal thickness data for assessment of clinically meaningful changes in uveitic macular edema.
The authors transformed central subfield thickness values into logarithmic equivalents for SD OCT. By defining the scale for $\operatorname{logSD}$ OCT a central subfield thickness of $250 \mu \mathrm{m}$ was assumed normal. A threestep change on the logSD OCT scale was equivalent to either doubling or halving of the central subfield thickness. Logarithmic transformation of SD OCT central retinal thickness provided a more normal distribution than central retinal thickness data alone and correlated positively with $\log$ MAR visual acuity. The correlation of $\log$ SD OCT with $\log$ MAR visual acuity suggested that changes in $\operatorname{logSD}$ OCT were clinically meaningful, objective, and a potentially useful measure for the evaluation of therapies targeting uveitic macular edema [87].

Swelling of Müller cells due to disturbed fluid transport has been described in states of macular edema [88, 89]. We believe, through combining UHR OCT and adaptive optics and thereby increasing image resolution, demonstration of in vivo morphological changes of Müller cells could unveil new information on the pathogenesis of uveitic macular edema.

Apart from macular edema, ERM, and vitreomacular traction, other macular pathological findings in patients with uveitis include macular hole and foveal atrophy (Figs. 6, 7) [90]. Forooghian et al. [91] characterized foveal atrophy in a heterogeneous group of patients with uveitis using HD-OCT images. The most common diagnoses associated with foveal atrophy were Behçet uveitis, serpiginous choroidopathy and sympathetic ophthalmia (13\%, each) followed by other posterior and panuveitis entities and intermediate uveitis. Using Cirrus HD-OCT, the authors defined foveal atrophy as a center point foveal thickness $<150 \mu \mathrm{m}$. The integrity of the photoreceptor layer was determined through examination of the IS/OS junction using the horizontal 5-line raster scan pattern. The status of the IS/OS junction was found to be associated with significant differences in mean visual acuity. The cause of foveal atrophy was multifactorial and included dysfunction and atrophy of the RPE and choroid, CME, macular ischemia secondary to occlusive retinal vasculitis, choroidal neovascularization, retinal detachment, and possibly antibody-mediated damage directed against photoreceptors. The authors suggested that careful observation of the IS/OS junction using HD-OCT could help to identify patients who are at risk for visual loss secondary to foveal atrophy. 

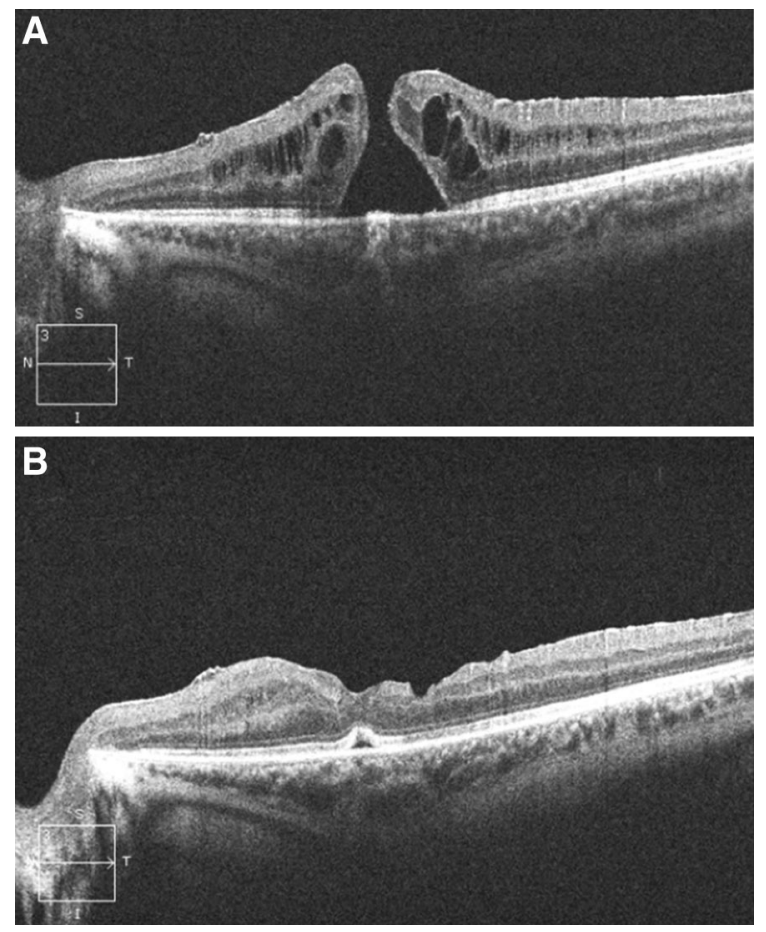

Fig. 6 High-definition SD OCT shows a full-thickness macular hole that developed secondary to cystoid macular edema in a patient with Behçet uveitis. Cystoid macular edema is noted at the edges of the macular hole (a). Closure of the macular hole with no interruption in the continuity of foveal tissue above the RPE layer after pars plana vitrectomy, membrane peeling, and gas-fluid exchange (b). SD OCT images of $9 \mathrm{~mm}$ scans were obtained using Cirrus HD-OCT with high-definition 5-line scan mode

\section{Vogt-Koyanagi-Harada disease}

Posterior segment manifestations of Vogt-KoyanagiHarada (VKH) disease are the earliest and extensively studied findings by OCT. Key OCT features of acutephase VKH disease are summarized in Table 1. While early studies involved the use of TD OCT, more recent studies focused on SD OCT findings of the disease. SD OCT of acute-phase VKH disease is shown in Fig. 8. Maruyama and Kishi [92] evaluated the cross-sectional image of serous retinal detachments using OCT 1 in eyes with VKH disease and reported that serous retinal detachments had two changes in structural patterns-a true serous retinal detachment and intraretinal fluid accumulation in the outer retina. They also reported observation of a thin reflective layer on the RPE and septa between the two types of fluid spaces, but they could not suggest what these structures represent. Parc

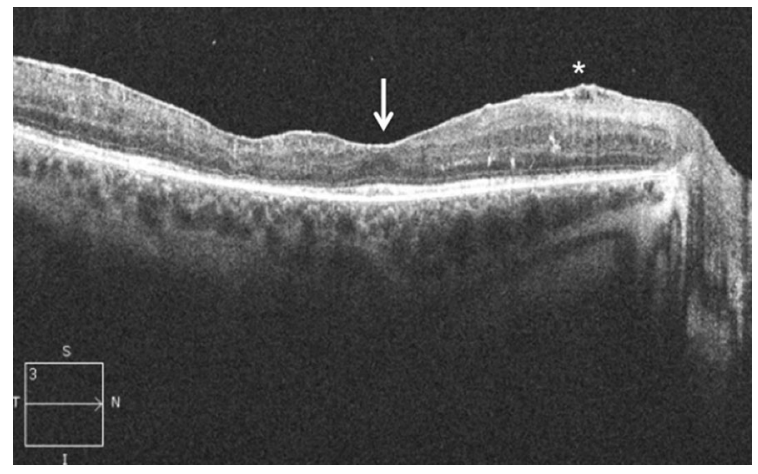

Fig. 7 Thinning and disruption of normal retinal architecture in a patient with Behçet uveitis. Loss of the IS/OS junction and OS/ RPE line (COST line), and thinning of the ONL. A thin hyperreflective line adhering to the inner retina represents an ERM formation; a prominent part of the ERM is indicated by the asterisk. Foveal depression is indicated by an arrow. SD OCT image of a $9 \mathrm{~mm}$ scan was obtained using Cirrus HD-OCT with high-definition 5-line scan mode

et al. [93] described the use of OCT to measure and monitor serous retinal detachment during the acute and chronic phases of VKH disease. OCT revealed an atypical serous retinal detachment with the presence of intraretinal edema with a hyporeflective area corresponding to fluid located between different layers of the neurosensory retina. Tsujikawa et al. [94] reported that the thin vertical walls of the large cystoid spaces within the subretinal fluid appeared as subfoveal yellowish round structures in eyes with VKH disease and assumed that cross-sectional images of OCT 2 showed intraretinal cystoid spaces. The authors hypothesized that the inner space was the subfoveal cystoid space and the outer space was the subretinal fluid. They also considered that the septa of the intraretinal cystoid spaces could represent subretinal fibrosis or a fibrin membrane, which is reportedly a feature in eyes with recurrent or chronic VKH disease. De Smet and Rao [95] interpreted peculiar subretinal septae seen by OCT as fibrinous strands. Yamaguchi et al. [96] reported similar findings on higher resolution OCT 3. They examined cross-sectional images of retinal detachments with multilobular dye pooling and monitored the subretinal structure after pulse corticosteroid therapy. Based on their observation that the subretinal septae dissolved immediately after pulse corticosteroid therapy, the authors concluded that the subretinal septae were composed of inflammatory products such as fibrin [96]. The rapid effect of pulse corticosteroid therapy on 
the serous retinal detachment observed at the acute uveitic phase of VKH disease had initially been reported by Yamanaka et al. [97] using a sequence of sectional images obtained by OCT. Other findings suggested, based on TD OCT scans, were the occurrence of fluid accumulation in the photoreceptor layer due to swelling of photoreceptors induced by acute inflammation and small focal limited RPE detachment at the level of severe dye leakage on FA $[98,99]$.

SD OCT further increased the understanding of retinal and choroidal manifestations of VKH disease. Using enhanced SD OCT images, Ishihara et al. [100] were able to clarify the pathologic features of multifocal serous retinal detachment that correspond to the multilobular pools of subretinal dye with dark rims on late-phase FA in eyes with acute VKH disease. The key findings were (1) uniform thickness of the membranous structure (named as septae in previous studies) forming the base of cystoid spaces; (2) continuity of the membranous structure with the outer photoreceptor layer of attached retina; (3) blurring around the cystoid spaces of the normally distinct line representing the IS/OS and continuity of the abnormal line with the highly reflective line within the membranous structure, suggesting that the membranous structure is a part of the outer photoreceptor layer; (4) intraretinal split in the outer photoreceptor layer that overlies the IS/OS line in the vicinity of the cystoid spaces in about half of the cases; and (5) a mean distance from the line representing the ELM to the anterior border of cystoid spaces in the central fovea that is much shorter than the length for the ISs/OSs indicating a separation of all or part of the OSs in this region. Based on OCT findings, the authors indicated that the OS becomes detached from the photoreceptor layer as intraretinal fluid accumulates; thereafter, inflammation causes fibrin to precipitate in the fluid space within the outer photoreceptor layer and acts as glue between the OSs and forms the membranous structure and the cystoid spaces characteristic of acute VKH disease. As corticosteroid therapy dissolves the fibrin, unbound pieces of the OSs have also been shown to collapse onto the RPE, appearing as granular structures on OCT.

Ikewaki et al. [101], using 3D OCT-1000 (Topcon, Tokyo, Japan), suggested that the initial visual acuity was significantly worse in $\mathrm{VKH}$ patients with a higher serous retinal detachment and that the initial visual acuity was not significantly correlated with the sensory retinal thickness. Another important outcome reported by the authors was the persistence of serous retinal detachment even after final visual acuity had been reached. Based on this finding, the authors concluded that the SD OCT parameters could be a reliable real-time indicator of the severity of inflammation and the effectiveness of treatment.

Gupta et al. [102] used SD Cirrus HD-OCT to study the structural alterations in the retina and RPE corresponding to the choroidal striations seen on FA in the acute uveitic phase of VKH disease. The occurrence of choroidal folds was initially demonstrated by Wu et al. [103] using TD OCT. Gupta et al. [102] reported that areas of serous RD were found to be adjacent to the large undulations on the 3D RPE map in the acute phase. The authors assumed that these undulations resulted from thickened choroid infiltrated with inflammatory cells that pushes the RPE forward, resulting in the formation of an undulation (Fig. 8). The troughs of these undulations corresponded to the choroidal striations seen in acute-stage VKH disease and are seen as hypo-fluorescent lines on FA. The small bumps seen on the surface of the undulation corresponded to the areas seen typically on FA as multiple pinpoint leakage sites, which result in late pooling of dye in the subretinal space [102]. The presence of undulations of RPE was also confirmed by Ishihara et al. using enhanced SD OCT and by Zhao et al. [100, 104] demonstrating 3D reconstructions of choroidal folds by using the 3D OCT-1000 (Topcon, Tokyo, Japan) device. Gupta et al. [102] also observed thickening or irregularity of the IS/OS junction in all eyes with acute VKH disease. The authors hypothesized that involvement of the photoreceptor in the acute phase could explain the delayed visual recovery. This finding was earlier attributed to the delayed regeneration of foveal cone photopigmentation despite the normal OCT scan results [105].

Vasconcelos-Santos et al. [106] studied the RPE and the outer retina in chronic VKH disease using FAF imaging and SD OCT. No FAF abnormality was reported corresponding to the sunset glow fundus. However, hypoautofluorescent lesions were associated with RPE loss and outer retinal involvement on SD OCT, and hyperautofluorescent lesions were associated with focal RPE hyperplasia and variable involvement of outer retina on SD OCT.

EDI SD OCT has been utilized to understand choroidal changes in VKH disease. EDI SD OCT 
Table 1 Key OCT features of Vogt-Koyanagi-Harada disease

\section{Acute phase}

Outer retina:

Subretinal fluid (serous retinal detachment) [92-94, 100]

Intraretinal cystoid spaces in the outer retina [92-94, 100]

Membranous structures (septae), that form the base of the intraretinal cystoid spaces, composed of inflammatory products such as fibrin that dissolve after intravenous pulse corticosteroid therapy [94-97, 100]

Separation of photoreceptor outer segments from photoreceptors in the vicinity of cystoid spaces [100]

Blurring of the photoreceptor IS/OS junction line around the cystoid spaces [100]

Thickening and irregularity of the IS/OS junction line [102]

Choroid and RPE:

Choroidal thickening leading to RPE undulation on the 3D RPE map and subretinal fluid accumulation [100, 102-104]

Troughs of choroidal/RPE undulations correspond to hypofluorescent lines on fluorescein angiography [102]

Small bumps on the surface of RPE undulations corresponding to multiple pinpoint leakage sites leading to dye pooling in the subretinal space on fluorescein angiography [102]

Massive inflammatory infiltration causing compression and nonperfusion of small choroidal vessels in the inner choroid leading to a reduction in hyperreflective dots corresponding to cross-sectional views of parallel-lying pericapillary arterioles and venules [109]

Subfoveal choroidal thickening [109, 111]

Inner retina:

Increased inner retinal thickness measured from ILM to IPL [112]

\section{Recovery phase}

Intact retinal architecture in eyes with sunset glow fundus [106]

Peripapillary atrophy exhibits thinning of RPE/BM (RPE atrophy) and loss of the IS/OS junction line, ELM and ONL with corresponding hypoautofluorescence on FAF, hyperfluorescence on FA (window defect), and hypofluorescence on ICGA (choriocapillaris nonperfusion) [106]

Nummular and/or irregular foci with decreased autofluorescence on FAF show thinning of the RPE/BM (RPE atrophy) and variable involvement of the outer retina ranging from sparing of the IS/OS junction line, ELM, and ONL to selective loss of one or more of these layers. FA and ICGA disclose corresponding hyperfluorescence (window defect) and hypofluorescence (choriocapillaris nonperfusion), respectively [106]

Atrophic scars with pigmentation show thickening of RPE/BM (RPE hyperplasia or hypertrophy) and variable involvement of the outer retina. FAF shows corresponding hypoautofluorescence. FA and ICGA reveal blockage of fluorescence [106]

Fundus lesions with increased autofluorescence (proliferation of RPE) manifesting as pigmented patches, irregular tracks and subretinal fibrosis show thickening of RPE/BM (RPE hyperplasia) usually with an intact outer retina. FA discloses corresponding hypofluorescence (blockage) [106]

Reduction in hyperreflective dots in the inner choroid due to shrinkage and dropout of small choroidal vessels caused by stromal scarring [109]

Normalization of subfoveal choroidal thickness [109, 111]

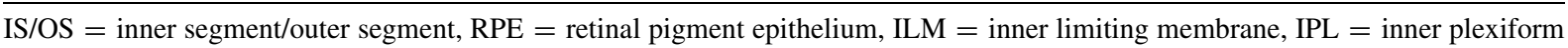
layer, $\mathrm{BM}=$ Bruch membrane, $\mathrm{ELM}=$ external limiting membrane, $\mathrm{ONL}=$ outer nuclear layer, $\mathrm{FAF}=$ fundus autofluorescence, $\mathrm{FA}=$ fluorescein angiography, ICGA = indocyanine green angiography

features of acute VKH disease are shown in Fig. 9. It is known that the retinal findings observed in $\mathrm{VKH}$ disease are secondary to choroidal inflammation [107, 108]. Fong et al. [109] used EDI SD OCT to examine the choroid of $\mathrm{VKH}$ patients in the acute and convalescent stages and to compare the findings with control subjects. The authors observed multiple hyperreflective dots in the inner choroid that increased in number towards the Bruch membrane in normal control eyes and speculated that these hyperreflective dots represent cross-sectional views of parallel-lying pericapillary arterioles and venules. EDI SD OCT highlighted a reduction of inner choroidal hyperreflective foci in VKH patients in the acute and convalescent stages of the disease (Fig. 9). This finding was attributed to the compression and nonperfusion of small choroidal vessels by massive infiltration of inflammatory cells and granuloma in the acute 

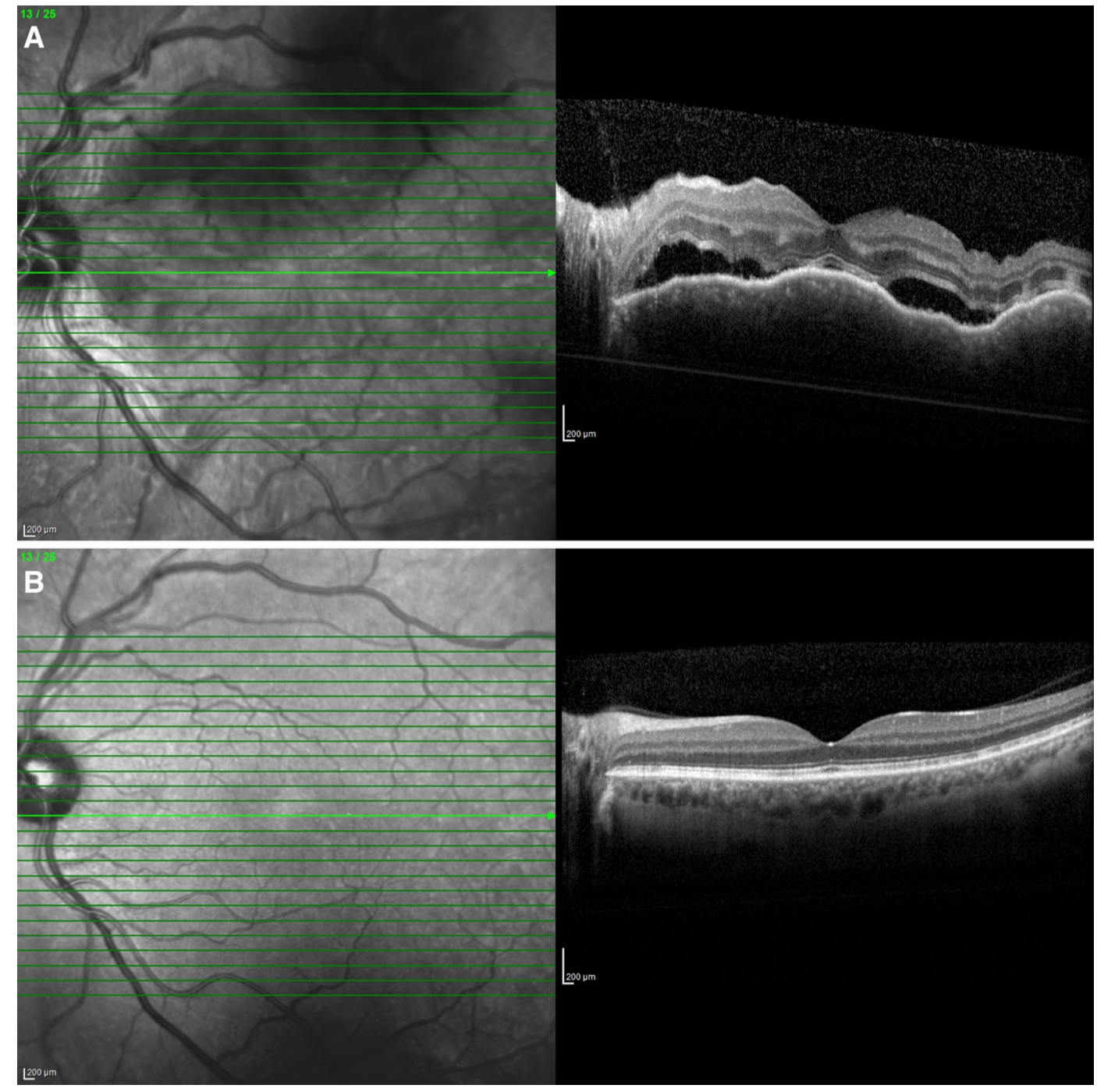

Fig. 8 High-resolution SD OCT shows serous retinal detachment and choroidal thickening leading to RPE undulation in the left eye of a patient with Vogt-Koyanagi-Harada disease in the acute uveitic phase (a). OCT using the autorescan feature shows resolution of the serous retinal detachment and RPE undulations

stage. Reduction of hyperreflective foci in the convalescent stage was interpreted as shrinkage and dropout of small choroidal vessels caused by stromal scarring. The authors also pointed to the possible presence of subclinical inflammation affecting the inner choroid in a large proportion of VKH patients despite high-dose immunosuppressive agents. This had previously been suggested by Herbort et al. [110]. The mean choroidal thickness in the acute phase was measured as $424 \mu \mathrm{m}$ as opposed to $287 \mu \mathrm{m}$ in control subjects. There was a statistically significant increase in choroidal thickness in the left eye of the same patient after high-dose systemic corticosteroid treatment (b). SD OCT image of a $9 \mathrm{~mm}$ scan was obtained using Heidelberg Spectralis OCT with a custom scan acquisition protocol of up to 25 raster lines of $9 \mathrm{~mm}$ scan length with automatic real-time imaging set at 25 images

in the acute phase compared with that in the convalescent phase by $151 \mu \mathrm{m}$ and compared with normal by $137 \mu \mathrm{m}$. The thickening returned to normal in the convalescent phase [109].

The subfoveal choroidal thickness in acute-phase VKH disease and its change with treatment has also been studied by Maruko et al. [111] using EDI SD OCT. The mean choroidal thickness in acute-phase VKH was measured as $805 \mu \mathrm{m}$ at baseline and was much thicker than those reported for normal eyes (Fig. 9). Maruko et al. [111] showed that baseline 
subfoveal choroidal thickness decreased dramatically after corticosteroid treatment by $14 \%$ at day $1,35 \%$ at day 3,49\% at day 7, and $58 \%$ at day 14 in patients with acute VKH disease. The serous retinal detachment decreased concurrently. By one month after initiation of corticosteroid treatment, the choroidal thickness was normal and the serous retinal detachment resolved.

Yamamoto et al. [112] evaluated inner retinal changes in patients with acute- and convalescentphase VKH disease using the Spectralis HRA and SD OCT device (Heidelberg Engineering). Inner retinal thickness was measured from the ILM to the inner plexiform layer (ILM-IPL), and RNFL thickness was measured at 1.5 and $2.0 \mathrm{~mm}$ along the outer border of the IPL from the central fovea at nasal, temporal, superior, and inferior points by enhanced SD OCT. Eyes with central serous chorioretinopathy (CSC) were evaluated as controls. In a previous study by Gupta et al. [102], the retina inner to ELM was found to have normal architecture and the disease to be confined mainly to the OS between the ELM and RPE in acute phase VKH disease. In contrast, Yamamoto et al. [112] showed that the ILM-IPL thickness in acute-phase VKH disease was significantly greater than that in the convalescent phase at each point of the retina. An intraretinal cystoid space was not observed. The thickness of the RNFL was significantly larger in the nasal quadrant. FA did not reveal any leakage from the retinal vasculature. In CSC patients no differences were seen in ILM-IPL thickness between the acute and convalescent phases at any point. Clinical implications of this finding remain to be identified.

\section{Sympathetic ophthalmia}

Gupta et al. [113] analyzed six consecutive patients diagnosed with sympathetic ophthalmia. All patients underwent FA and Cirrus HD-OCT scan and were treated with high-dose systemic corticosteroids. The
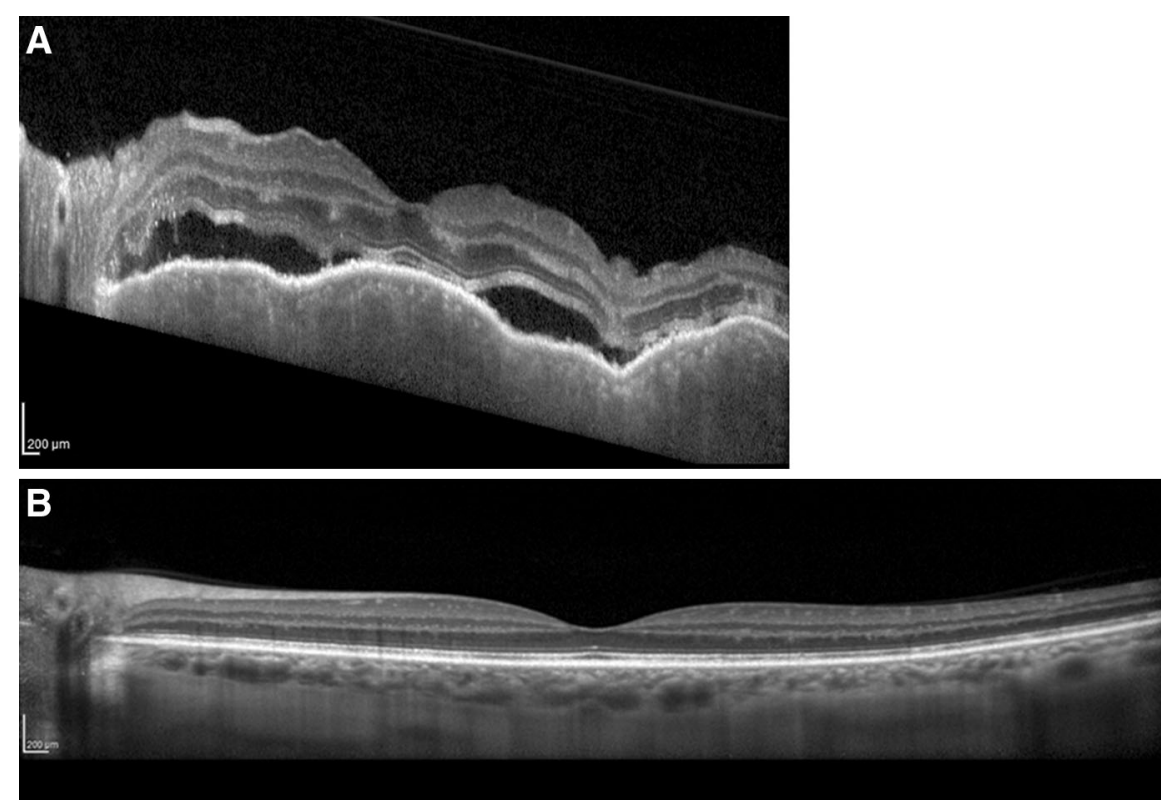

Fig. 9 EDI OCT shows massive inflammatory infiltration causing subfoveal choroidal thickening and compression and nonperfusion of small choroidal vessels in the inner choroid leading to a reduction in hyperreflective dots corresponding to cross-sectional views of parallel-lying pericapillary arterioles and venules in the left eye of a patient with Vogt-KoyanagiHarada disease in the acute uveitic phase (a). EDI OCT image of a $9 \mathrm{~mm}$ scan was obtained using Heidelberg Spectralis OCT with a custom scan acquisition protocol of up to 25 raster lines of
$9 \mathrm{~mm}$ scan length with automatic real-time imaging set at 25 images EDI OCT shows normalization of choroidal thickness and reappearance of hyperreflective dots corresponding to crosssectional views of parallel-lying pericapillary arterioles and venules in the left eye of the same patient after high-dose systemic corticosteroid treatment (b). EDI OCT of a $9 \mathrm{~mm}$ line scan centered at the fovea was obtained using Heidelberg Spectralis OCT with automatic real-time image averaging set at 100 images 
study showed photoreceptor involvement in the acute stage, which was reversible in all sympathizing eyes in the early stage of the disease. At baseline, the retina inner to ELM did not show any remarkable structural alteration and there was serous retinal detachment with elongation of the photoreceptors in the OS demarcated by the ELM and RPE lines. Although the RPEchoriocapillaris complex could be delineated, there was disruption of the continuity of the IS/OS and OS/ RPE (COST) junction lines. None of the eyes showed intraretinal cystic spaces at presentation. Two eyes showed hyperreflective bands indicating fibrinous septae within the serous detachment. A single-layer RPE scan map of two eyes showed undulations and bumps on the RPE surface with one patient showing the presence of choroidal striations on ophthalmoscopy. The undulations were seen adjacent to the serous retinal detachment on the raster line scans. In the remaining four eyes, serous detachment did not allow interpretation of the single-layer 3D RPE map. Visual acuity recovered gradually over the following two weeks after systemic corticosteroid therapy and coincided with the resolution of serous retinal detachment as well as restoration of the photoreceptors and IS/OS junction line as seen on OCT scans. On systemic corticosteroid therapy, the single-layer RPE scans showed a reduction of the dome-like undulations within 48 h. Repeat OCT scans performed at 12 weeks showed complete resolution in all six patients with a normal single-layer RPE surface map.

TD OCT scans of Dallén-Fuchs nodules were analyzed in one patient with sympathetic ophthalmia [114]. Dallén-Fuchs nodules appeared as discrete nodules at the level of RPE and were associated with mild shadowing and overlying detachment of the neurosensory retina.

Acute posterior multifocal placoid pigment epitheliopathy

Key OCT features of acute posterior multifocal placoid pigment epitheliopathy (APMPPE) are summarized in Table 2. Lofoco et al. [115] described the OCT findings in one patient with APMPPE. An acute-phase OCT scan revealed a mild hyperreflective area above the RPE in the photoreceptor layer. In the later phases, OCT showed a nodular hyperreflective lesion on the plane of the RPE with mild underlying backscattering. Lim et al. [116] reported on another patient with APMPPE where an OCT scan was performed through an acute lesion and then repeated through the same lesion 12 months later. The initial OCT scan showed marked anterior displacement of both the neural retina and the outer reflective band. The subsequent OCT scan revealed resolution of the prior displacement, increased reflectance of the outer reflective band and mild disruption of the outer retinal layers. These changes were found to be compatible with the enlargement of the underlying choroid by an inflammatory infiltrate that is associated with choroidal vasculitis.

Scheufele et al. [117] used UHR OCT to define the morphological changes occurring in APMPPE in more detail in three patients-one with acute and two with healed lesions. In the three cases, UHR OCT demonstrated early and late photoreceptor disruption which had not been demonstrated previously in this disease. In the acute setting, areas of increased backscattering were seen in the outer neural layer, probably representing early degenerative changes of the photoreceptors. Simultaneous FA and indocyanine green angiography (ICGA) demonstrated hypofluorescence of these lesions. The authors speculated that choroidal fluorescence may be blocked by the inflamed or damaged photoreceptor cell bodies seen concurrently on UHR OCT or alternatively, due to choroidal ischemia, which would also result in ischemia of the RPE and outer retina and could account for the photoreceptor and RPE disruption seen on the UHR OCT. RPE disruption was demonstrated after the lesions healed. Garg and Jampol observed similar lesion backscattering on TD OCT in a patient with APMPPE and serous macular detachments [118]; however, UHR OCT demonstrated more clearly that these lesions were located in the outer retina [117]. The presence of serous macular detachment and intraretinal fluid has been further demonstrated in APMPPE [119-121]. Birnbaum et al. [119] showed the presence of macular subretinal fluid on OCT in two of eight patients with APMPPE. Lee et al. [120] reported on a patient with acute APMPPE mimicking VKH disease. The authors demonstrated pockets of macular and peripapillary subretinal fluid as well as compartmentalized subretinal fluid with detachment of the overlying neurosensory retina. Presence of hyperreflective subretinal deposits within the fluid; relative preservation of the RPE line; disruption of the IS/OS junction line, ELM and outer nuclear layers (ONLs); and significant choroidal thickening was 
Table 2 Key OCT features of acute posterior multifocal placoid pigment epitheliopathy

\section{Acute phase}

Photoreceptor disruption leading to hyperreflectivity in the photoreceptor layer above RPE and underlying backscattering [115$118,122-125]$

Alterations in the outer retinal layers including the external limiting membrane, IS/OS junction, and OS/RPE (COST) junction line [120]

Subretinal fluid or large intraretinal cysts in the outer nuclear layer in some patients [118-121]

\section{Recovery}

Photoreceptor atrophy, lack of reconstitution of the IS/OS junction and OS/RPE lines, and disruption of RPE [116, 117, 125]

$\mathrm{RPE}=$ retinal pigment epithelium, OS = outer segment, COST $=$ cone outer segment tip, IS/OS = inner segment/outer segment

documented in the acute phase. Over the follow-up there was early resolution of subretinal fluid and deposits, loss of OS/RPE (COST), IS/OS, and ELM lines, as well as significant thinning of the ONL. The healing process ended with reorganization and normalization of outer retinal structures with partial reappearance of the IS/OS line and ELM, reorganization of the OS/RPE junction (COST) line and normalization of ONL. Montero et al. [121] reported on the presence of large intraretinal cysts in the ONL consistent with intraretinal edema in a patient with acute APMPPE. There was early resolution of the macular cysts that was associated with appearance of a hyperreflective line corresponding to the area previously occupied by the retinal cyst and increased backscatter suggesting initial retinal pigment epithelium (RPE) disruption. SD OCT showed normalization of the gross anatomy of the fovea at 3 months; however, macular thinning and disruption of the RPE and IS/OS junction lines were observed.

Other studies have also confirmed early photoreceptor and RPE disruption occurring as a consequence of either primary retinal pigment epitheliitis or as a consequence of ischemia involving the choroidal capillaries [122-125]. Alterations in the outer retinal layers including the ELM, IS/OS junction, and OS/ RPE (COST) lines were also confirmed. Cheung et al. [125] reported that despite visual recovery there was photoreceptor atrophy and lack of reconstitution of the IS/OS junction and OS/RPE (COST) lines in resolved APMPPE and that this disturbance even involved areas that were unaffected both on ophthalmoscopy and OCT in the acute phase.

Optical coherence tomography findings of one patient with relentless placoid chorioretinitis (RPC) were defined by Amer and Florescu [126]. RPC has been defined as a unique entity with atypical clinical findings and course resembling both APMPPE and serpiginous choroidopathy. TD OCT during the active stage of the RPC showed foveal subretinal fluid and pigment epithelium detachment with hyperreflectivity of the inner and outer retinal layers, suggesting a chorioretinal pathogenetic process. Similar to active lesions in APMPPE and serpiginous choroidopathy OCT showed hyperreflectivity of outer retinal layers in active RPC.

Acute zonal occult outer retinopathy

Key OCT features of are defined in Table 3. Li and Kishi [127] examined seven eyes with acute zonal occult outer retinopathy (AZOOR) in five patients using TD OCT and found loss of the IS/OS junction in those with visual field defects and loss of ONL in two eyes. Photoreceptor atrophy corresponding to dysfunctional retina has been further demonstrated by TD and en-face OCT in acute AZOOR [128, 129].

Spaide et al. [130] defined the SD OCT features in a group of patients diagnosed with AZOOR complex diseases. The study involved patients with AZOOR, multiple evanescent white dot syndrome (MEWDS), and multifocal choroiditis and panuveitis (MCP). Common to all of these entities is the occurrence of an enlarged blind spot. In all patients with blind spot enlargement there were peripapillary IS/OS junction line defects. Peripapillary IS/OS line defects were not encountered in eyes that did not have visual field defects. Patients with AZOOR had blind spot enlargement with associated scotomata and SD OCT evidence of loss of the photoreceptor OSs and cell bodies. At the junctional areas between preserved and severely damaged retina were areas of loss of the IS/OS line alone. In patients with AZOOR, outer retinal problems were more severe and were associated with loss of the IS/OS line and ONL and with thinning of the INLs 
without visible correlates in the fundus. Treatment of AZOOR patients with immunomodulation was associated with improvement in visual field analysis and with restoration of the IS/OS junction in areas where the ONL appeared to have a relatively normal thickness. However, there was no visual field or anatomic improvement in regions with ONL loss. This implies that in AZOOR, loss of OSs, which are defects occurring as a consequence of injury to photoreceptor cellular processes, may show signs of reconstitution of the OSs and hence the IS/OS junction line, whereas injury resulting in actual loss of photoreceptors does not.

Fujiwara et al. [131] determined FAF and SD OCT findings in patients with AZOOR and reported RPE abnormalities as detected by FAF. The thickness of the choroid was normal as detected by EDI SD OCT. There was abnormality of both the OS and the thickness of the photoreceptors in general. In the overlying areas of photoreceptor loss, there were abnormalities in the normal lamellar design of the retina, although the thickness of the inner retina was not specifically abnormal.

Additional changes in AZOOR determined by SD OCT involve the OS/RPE (COST) line. Tsunoda et al. [132] first reported indistinctiveness of the OS/RPE (COST) line that was evident through the entire follow-up. There was also absence of the foveal bulge. The IS/OS junction was preserved. The authors suggested that the cone photoreceptor OS dysfunction could be initially reflected by the indistinctiveness of the OS/RPE (COST) line and the absence of foveal bulge and that abnormalities in the IS/OS junction may follow these changes in the more advanced stages. So et al. [133] also reported loss of the OS/RPE (COST) line in acute phase AZOOR. In the acute phase, selective loss of the IS/OS and the OS/RPE (COST) lines corresponded with reduced visual acuity and mfERG potentials in areas of visual field defects. In the recovery phase, disappearance of the scotoma was associated with improvement in visual acuity and recovery of mfERG. The absence of the OS/RPE (COST) line in the recovery phase appeared as a subclinical sign. Another finding that has been shown in convalescent AZOOR is a decrease in the retinal thickness and has been attributed to shortening of photoreceptors and ONL and thinning of the INL [134].

One study combined SD OCT with adaptive optics scanning laser ophthalmoscopy (AOSLO) to evaluate four patients with reduced visual function and in whom retinal changes assessed by standard techniques were insufficient to explain the visual abnormalities [135]. Adaptive optics, when used with an ophthalmoscope, allows for direct imaging of the cone photoreceptor mosaic in vivo. Macular cone structure as assessed by AOSLO in AZOOR revealed heterogeneity with normal mosaic of cones, regions of cone loss, and alterations in cone structure. SD OCT showed a disrupted IS/OS junction in regions with

Table 3 Key OCT features of acute zonal occult outer retinopathy

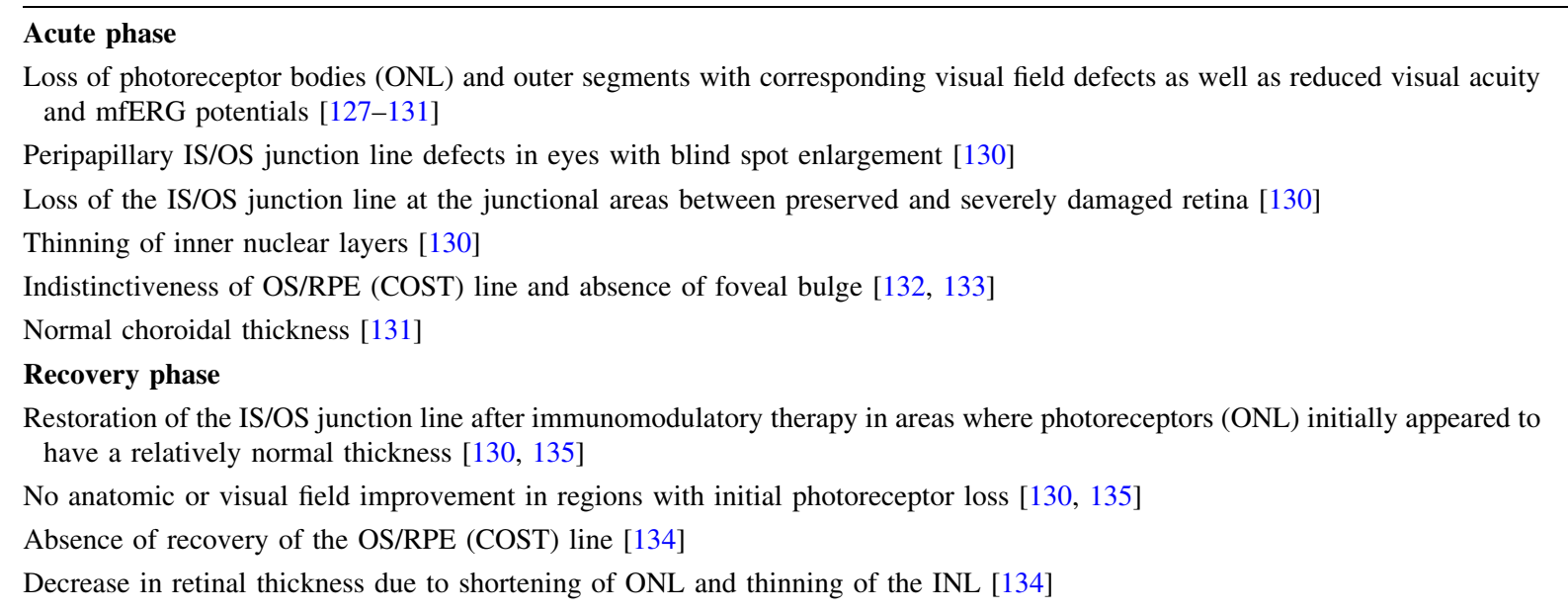

$\overline{\mathrm{ONL}}=$ outer nuclear layer, $\mathrm{mfERG}=$ multifocal electroretinography, IS/OS $=$ inner segment/outer segment, OS/RPE $=$ outer segment/retinal pigment epithelium, COST $=$ cone outer segment tip, INL $=$ inner nuclear layer 
reduced cone reflectivity; reduced reflectance of the IS/OS junction in regions with cone loss; relative thinning of the ONL and ganglion cell layer, and intact ELM-OS/RPR (COST) interval where cone mosaic was normal; and loss of the IS/OS junction with thinning of the ONL in regions with abnormal cone coverage and spacing on AOSLO.

Vance et al. [136] described SD OCT features of acute macular neuroretinopathy in four patients. Acute macular neuroretinopathy is also grouped with AZOOR complex diseases. All patients showed ophthalmoscopically dark areas near the fovea that corresponded to focal IS/OS abnormalities by SD OCT imaging. Over the follow-up ranging from 1 to 5 months, IS/OS defects resolved in two patients and improved in the remaining two patients, but all patients continued to manifest focal ONL thinning.

\section{Multiple evanescent white dot syndrome}

Key OCT features of MEWDS are described in Table 4. OCT has shed new light on the confusing pathogenesis of MEWDS. The first case report using TD OCT showed a disrupted IS/OS junction line at the macula that recovered 21 weeks later [137].

Spaide et al. [130] observed widespread loss of the IS/OS junction even in regions between the visible white spots during the acute phase of MEWDS. By four months after onset, spontaneous concurrent improvement occurred in both blind spot enlargement in the visual field and the IS/OS line disruption on the SD OCT scans. A disturbance of the IS/OS junction in patients with MEWDS was also described by Nguyen et al. [138]. Apart from IS/OS junction disturbance observed in all eyes, UHR OCT showed thinning of the ONL in one patient with recurrent disease [138].

$\mathrm{Li}$ and Kishi [139] studied seven patients with MEWDS using SD OCT and full-field EFG (ffERG) and mfERG. SD OCT showed a disrupted IS/OS line as the main feature of MEWDS. In acute MEWDS, the IS/OS line was diffusely or spottily disrupted. The disrupted IS/OS line recovered quickly and achieved continuity 4.5 weeks to 6 months after disease onset. Figure 10 shows disruption and recovery of the IS/OS junction line in MEWDS. No abnormalities were noted in the ONL and RPE during follow-up. The authors concluded that unlike AZOOR where loss of photoreceptor cell bodies results in lack of reconstitution of the outer retina, disruption of the OSs but intact photoreceptor cell bodies in MEWDS results in almost complete recovery of the photoreceptor OSs and hence the IS/OS line [139]. The reduced ffERG and mfERG amplitudes during the acute stage, which recovered with restoration of the IS/OS line, supported the presumption that the main lesion is associated with damage to the photoreceptor OS, but not the RPE or choriocapillaries. A restoration of photoreceptor OS length along with recovery of FAF and microperimetry has been shown to occur with disease recovery by others [140].

It has also been suggested that disruption of the IS/ OS line is associated with RPE inflammation. RPE inflammation could result in swelling of RPE cells leading to the appearance of an intact but thickened RPE line or separation of RPE from the Bruch membrane on OCT. The inflammation in RPE in turn could lead to an acute interruption in the orientation of photoreceptor OSs, causing attenuation of OCT signal from the IS/OS junction line as the photoreceptor OSs become misaligned [141]. Therefore, disruption of the IS/OS junction line in MEWDS may represent an artifact induced by RPE swelling.

Although SD OCT studies pointed to a disease process involving the photoreceptor OSs and the IS/ OS junction with no pathology involving any other retinal layer or the inner choroid in patients with acute MEWDS [142, 143], one study showed a subfoveal choroidal thickening [144]. Aoyagi et al. [144] studied the subfoveal choroidal thickness using SD OCT in two patients with acute MEWDS. In the acute phase there was a thickening in the subfoveal choroid in the involved eyes compared to the fellow eyes of both patients. In the convalescent phase there was a decline in the choroidal thickness not only in the involved eye but in both eyes. The authors suggested that the choroidal thickening could point to an inflammatory reaction involving the choroid. Choroidal involvement has also been suggested based on TD OCT findings of focal subretinal dome-like material accumulation and increased reflectivity of choroid at the same point in one patient with acute MEWDS [145].

Multifocal choroiditis and panuveitis

Spaide et al. [130] revealed loss of the IS/OS line over chorioretinal scars in patients with MCP. There was also SD OCT evidence of absence of the IS/OS line 
Table 4 Key OCT features of multiple evanescent white dot syndrome

\section{Acute phase}

Widespread, even in regions between the visible white spots, loss of the IS/OS junction (diffusely or spottily) due to loss of photoreceptor outer segments with retaining of normal photoreceptor bodies [130, 137-139, 142, 143]

Normal retinal pigment epithelium [139]

Subfoveal choroidal thickening [144, 145]

\section{Recovery phase}

Restoration of the IS/OS junction line within 4.5 weeks to 6 months $[130,137,139,140]$

IS/OS $=$ inner segment/outer segment

and thinning of the ONL over and in the immediate areas surrounding scarring from old choroidal neovascular membrane. Patients with MCP who had blind spot enlargement also had corresponding contiguous regions of the IS/OS junction loss around the optic nerve head even in ophthalmoscopically normal areas between visible chorioretinal scars [130].

Yasuno et al. [146] used a custom-built HP OCT to assess the retina and the choroid of patients with MCP. Both B-scans and en face projections were obtained. HP OCT scans revealed the following characteristic properties of the lesion sites - thinning of the retina, destruction of the retinal layers, and disappearance IS/ OS junction line. Due to the high penetration of this OCT system, the following characteristic properties of the choroid were also observed: localized thinning of the choroid, occlusion of the choroidal vessels, and localized hyperreflectivity that may represent hyperpigmentation of the choroid. The choroidal pathologies visualized by HP OCT were associated with the findings of color fundus photography, FA, and ICGA images. The authors concluded that HP OCT could be used as a noninvasive method to distinguish the stages of MCP progression.

\section{Punctate inner choroidopathy}

Optical coherence tomography has been used to document and assess the effect of treatment on choroidal neovascular membranes secondary to punctate inner choroidopathy (PIC) [147-149]. Stepien and Carroll [149] demonstrated SD OCT evidence of intraretinal fluid accumulation secondary to choroidal neovascular membrane and homogenous outer retinal thickening over chorioretinal lesions in association with recurrent inflammatory activity in a patient with recurrent PIC.
Serpiginous chorioretinitis and multifocal choroiditis

Van Velthoven et al. [150] described the en-face OCT and confocal ophthalmoscopic findings in active multifocal and serpiginous chorioretinitis shortly after the onset of symptoms. Apart from involvement of the outer retina and choroid, morphological information obtained by en-face OCT revealed that the process extended into the inner retina leading to a circumscribed, focal retinal thickening in the OCT C-scans in eyes with multifocal chorioretinitis. In serpiginous chorioretinitis, morphological information acquired revealed that acute-phase inflammation is limited to the deeper retinal and choroidal structures. Taken together, the OCT manifestations of multifocal and serpiginous chorioretinitis were different at the same stage of the pathophyisological process.

Vance et al. [151] compared the SD OCT findings of multifocal choroiditis with those of new-onset myopic choroidal neovascularization. The authors concluded that the acute lesions of multifocal choroiditis showed drusen-like material between the RPE and the Bruch membrane, vitreous cells, and localized choroidal hyperreflectivity below the RPE material. These OCT findings were not usually present in eyes with myopic choroidal neovascularization. The subretinal pigment epithelial material corresponded to acute lesions found on color photographs and FA. The subretinal pigment epithelial material and choroidal hyperreflectivity appeared to improve after treatment with anti-inflammatory or anti-vascular endothelial growth factor therapy. In contrast, SD OCT in the patients with myopic choroidal neovascularization showed a very thin choroid, a posterior staphyloma, and a type 2 (subretinal) neovascular pattern. Kotsolis et al. [152] using SD OCT technology concluded that 

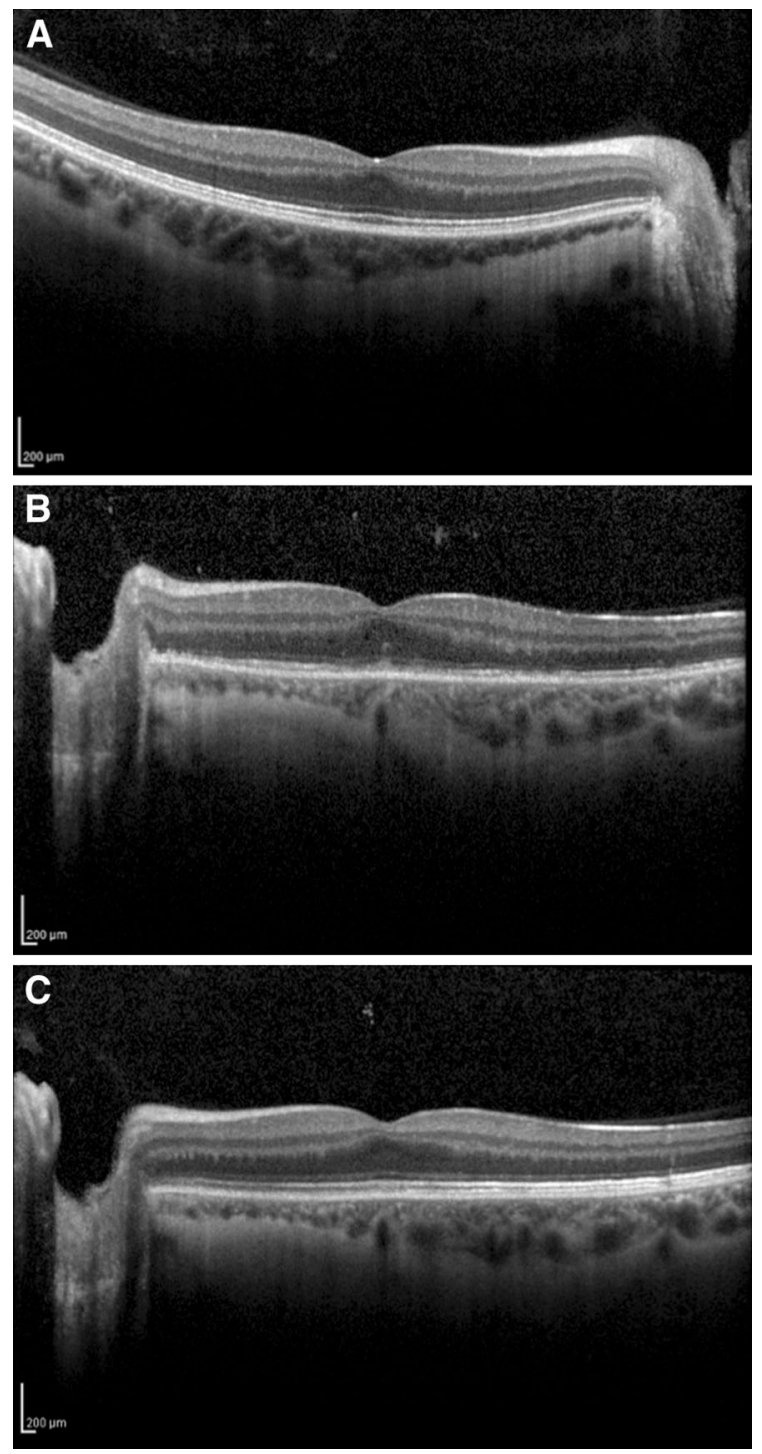

Fig. 10 High-resolution SD OCT shows an intact IS/OS line in the uninvolved right eye of a patent with multiple evanescent white dot syndrome (a). SD OCT shows a spottily disrupted IS/ OS junction line in the involved left eye of the same patient (b). Achievement of continuity and rapid recovery of the IS/OS junction line along with a partially recovered OS/RPE line (COST line) is observed eight weeks after disease onset (c). SD OCT images of a $9 \mathrm{~mm}$ scan were obtained using Heidelberg Spectralis OCT with a custom star-shaped scan acquisition protocol of $9 \mathrm{~mm}$ scan length with automatic real-time imaging set at 20 images

FA was superior to OCT in revealing choroidal neovascularization in patients with multifocal choroiditis and recommended the use of FA in imaging choroidal neovascular membranes in eyes with multifocal choroiditis. OCT has also been used to gather preoperative clues for submacular surgery for choroidal neovascular membrane removal. Eyes with neovascular membranes located anterior to and separated from the RPE that had an 'optically clear zone' underneath were the best candidates for surgical removal [153].

SD OCT technology has also been utilized to define retinal morphological changes in patients with serpiginous choroiditis $[154,155]$. In one study, FAF was evaluated along with SD OCT in a patient with recurrent serpiginous choroiditis. Active new lesions disclosed hyperautofluorescence in contrast to hypoautofluorescence in scarred lesions. OCT showed increased reflectance of the choroid and deeper retinal layers, along with disruption of the photoreceptor IS/ OS junction in both active and inactive lesions [155]. Figure 11 shows a high-definition SD OCT scan of a patient with serpiginous-like choroiditis.

\section{Birdshot retinochoroidopathy}

UHR OCT in a case with birdshot retinochoroidopathy revealed disorganization of inner retinal layers as well as photoreceptor and RPE atrophy [156]. Monnet et al. [157] used OCT 3 and FA to evaluate the macula in 80 patients with birdshot retinochoroidopathy. There was not good agreement between macular edema identified by OCT and FA. Decreased visual acuity was found to be related to abnormal macular thickness and loss of the IS/OS junction as determined by OCT and vascular leakage on FA. When compared with macular parameters, symptoms were related most closely to loss of the IS/OS junction line. There were no strong relationships between birdshot lesion characteristics and macular parameters. Birch et al. [158] used SD OCT technology to evaluate the macula in patients with birdshot retinochoroidopathy. Segmentation of retinal layers was performed using the software included with the Spectralis HRA and SD OCT device (Heidelberg Engineering). The results showed that total retinal thickness was reduced in all eyes and at all locations when compared to the normal control eyes. The reduction in the retinal thickness of the macula was associated with a reduction in the segment extending from the proximal border of the outer plexiform layer to the Bruch membrane-choroid interface reflecting the photoreceptors and RPE (Fig. 12). The inner retinal layer thickness was within 

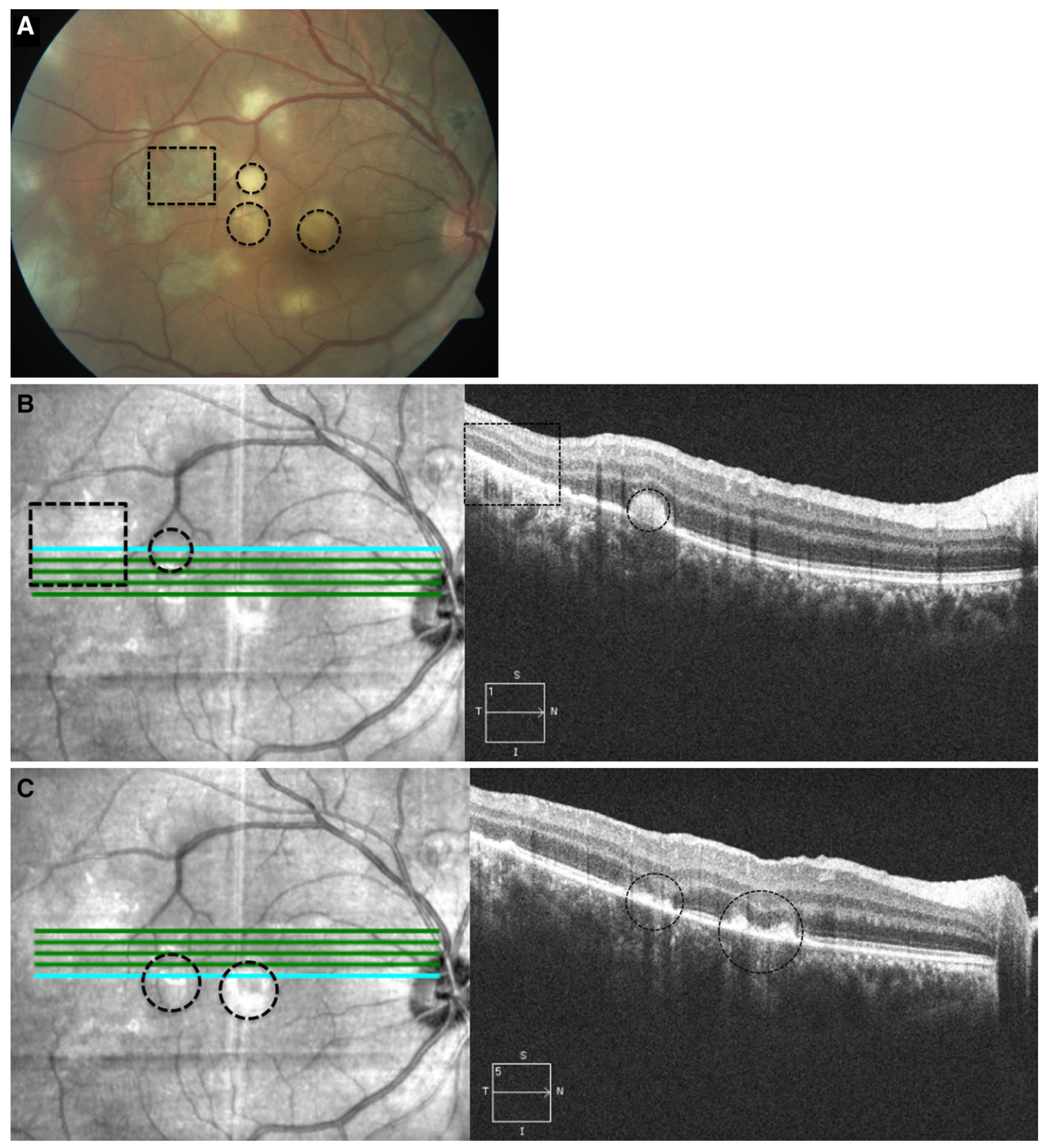

Fig. 11 Color fundus photograph of a patient with serpiginouslike choroiditis; dashed-line circles indicate active foci of choroiditis and dashed-line square bounders a choroiditis scar (a). SD OCT image of $9 \mathrm{~mm}$ scans were obtained using Cirrus HD-OCT with high-definition 5-line scan mode. LSO fundus image shows the location of scan line. OCT B-scan image shows a dome-shaped hyperreflective focus leading to shadowing of the underlying choroid. The ELM, IS/OS junction line, OS/RPE line (COST line) and RPE lines are indiscernible at the active lesion site (dashed-line circle). OCT B-scan reveals loss of ELM, IS/OS junction, and OS/RPE (COST) lines and focal choriocapillaris/choroidal relative hyperreflectivity at the choroiditis scar (dashed-line square) (b). LSO fundus image shows the location of scan line. OCT B-scan image shows domeshaped hyperreflective foci leading to shadowing of the underlying choroid. The ELM, IS/OS junction, OS/RPE (COST) and RPE lines are indiscernible at the active lesion sites (dashedline circles); the ONL is thinned and OPL is disfigured (c) 

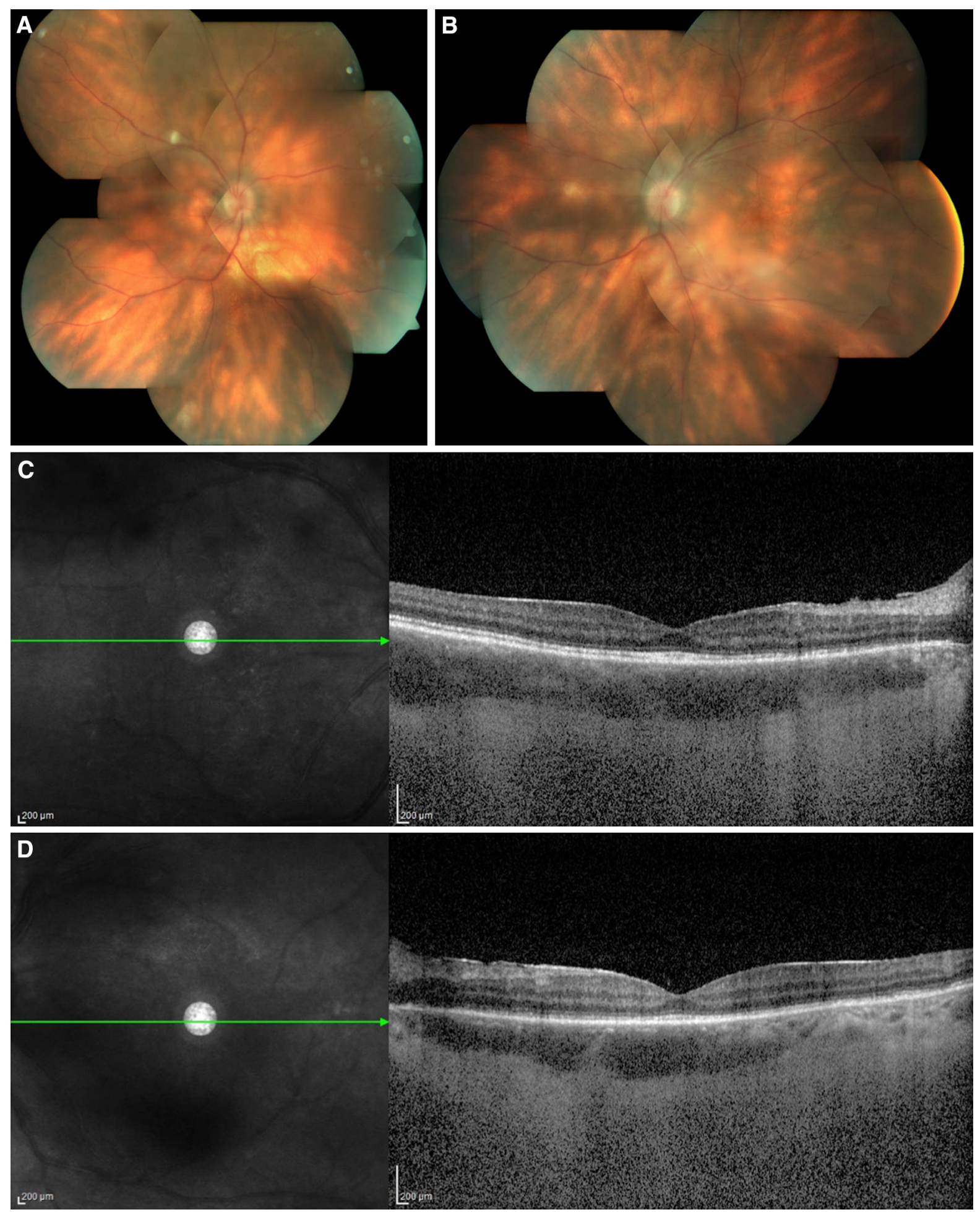

normal limits in most patients. Restoration of the retinal architecture following systemic immunomodulatory treatment in a case with birdshot retinochoroidopathy has also been reported using SD OCT [159]. SD OCT of birdshot retinochoroidopathy is shown in Fig. 12. 
4Fig. 12 Composite color fundus photograph of the right (a) and left eye (b) of a patient with birdshot retinochoroidopathy. SD OCT shows retinal thinning more prominent in the segment extending from the proximal border of the outer plexiform layer to the Bruch membrane-choroid interface reflecting the photoreceptors and RPE in the right (c) and left eye (d). OCT B-scan also shows disruption of the IS/OS junction line and loss of OS/RPE line (COST line). SD OCT of a $9 \mathrm{~mm}$ line scan centered at the fovea was obtained using Heidelberg Spectralis OCT with automatic real-time image averaging set at 25 images

\section{Behçet uveitis}

Optical coherence tomography was also utilized to understand pathological changes of the neurosensory retina in Behçet uveitis. Retinal vessels are the primary target of occlusive vasculitis that occurs in Behçet disease; however, one study suggested that there may be retinal thickening or normal retinal thickness as assessed by OCT in eyes with documented choroidal thickening on echography. The authors suggested primary choroidal vasculitis as the cause of choroidal thickening in eyes with Behçet uveitis [160]. Atmaca et al. [161] compared macular OCT findings with FA in 33 eyes of 18 patients with Behçet uveitis. Macular edema and thickening on TD OCT was evident in 10 eyes with variable pathological findings on FA. Nine eyes with variable pathological findings on FA were documented to have normal OCT scans. OCT showed a macular hole in one eye and the remaining 13 eyes had normal FA and OCT scans [161]. Takeuchi et al. [162] analyzed functional changes of the macula by microperimetry and structural changes by TD OCT during clinical remission of ocular inflammation in patients with Behçet uveitis. Visual acuity correlated with perimetric sensitivity but not with retinal thickness based on OCT. The duration of Behçet uveitis correlated with visual acuity and with retinal thickness, but not with perimetric sensitivity. No correlation was found between retinal thickness and the corresponding retinal sensitivity. The results suggested that visual acuity, retinal perimetric sensitivity, and retinal thickness decrease with increasing duration of Behçet uveitis. Among these factors, retinal perimetric sensitivity seemed to be relatively preserved. Unoki et al. [163] documented foveal changes during remission of Behçet uveitis using SD OCT. Visual acuity was significantly better in eyes with an intact IS/OS line than in eyes with an ill-defined IS/OS line. Male gender was more likely to have absence of the IS/OS line. Foveal thickness of the IS/OS intact eyes was significantly more than IS/OS absent eyes. Status of the ELM was not associated with changes in visual acuity.

A finding not previously described is the occurrence of RNFL defects in Behçet uveitis. Wedgeshaped defects in the peripapillary RNFL, corollary thinning detected by OCT, and corresponding visual field loss may occur in Behçet patients in the absence of central nervous system involvement or prior optic neuritis (Fig. 13). The neuroretinal rim is well preserved in these cases in contrast to the loss of neuroretinal rim associated with RNFL atrophy in glaucoma. These defects typically appear following resolution of retinal infiltrates at the posterior pole, suggesting an acute focal damage to the ganglion cell layer and the RNFL. The same clinical picture involving focal RNFL loss with a preserved neuroretinal rim has also previously been described in ocular toxoplasmosis [164].

\section{Ocular toxoplasmosis}

Key OCT features of ocular toxoplasmosis are summarized in Table 5. Changes in the retinal architecture and vitreoretinal interface in ocular toxoplasmosis have been analyzed using OCT technology. Oréfice et al. [165] examined the vitreoretinal morphology in patients with active punctate, focal, and satellite ocular toxoplasmosis lesions. On OCT 3 scans, retinal layers were hyperreflective at the active lesion site and some degree of retinal RPE-choriocapillaris/choroidal optical shadowing was seen in all patients. In general, the retina was thinned at the active lesion site in eyes with punctate lesions and thickened in eyes with focal and satellite lesions. When detected by OCT 3, the posterior hyaloid appeared thickened. While focally detached over punctate lesions, the posterior hyaloid was partially detached, but still attached to the lesion in focal and satellite lesions. Additional findings that were not detected on clinical examination included presence of DME, vitreomacular traction and maculoschisis. During follow-up, a decrease in retinal thickness and focal choriocapillaris/choroidal relative hyperreflectivity were observed at the former lesion site, and posterior vitreous detachment was documented in all patients.

Punctate outer retinal toxoplasmosis (PORT) is an atypical clinical presentation of ocular toxoplasmosis 

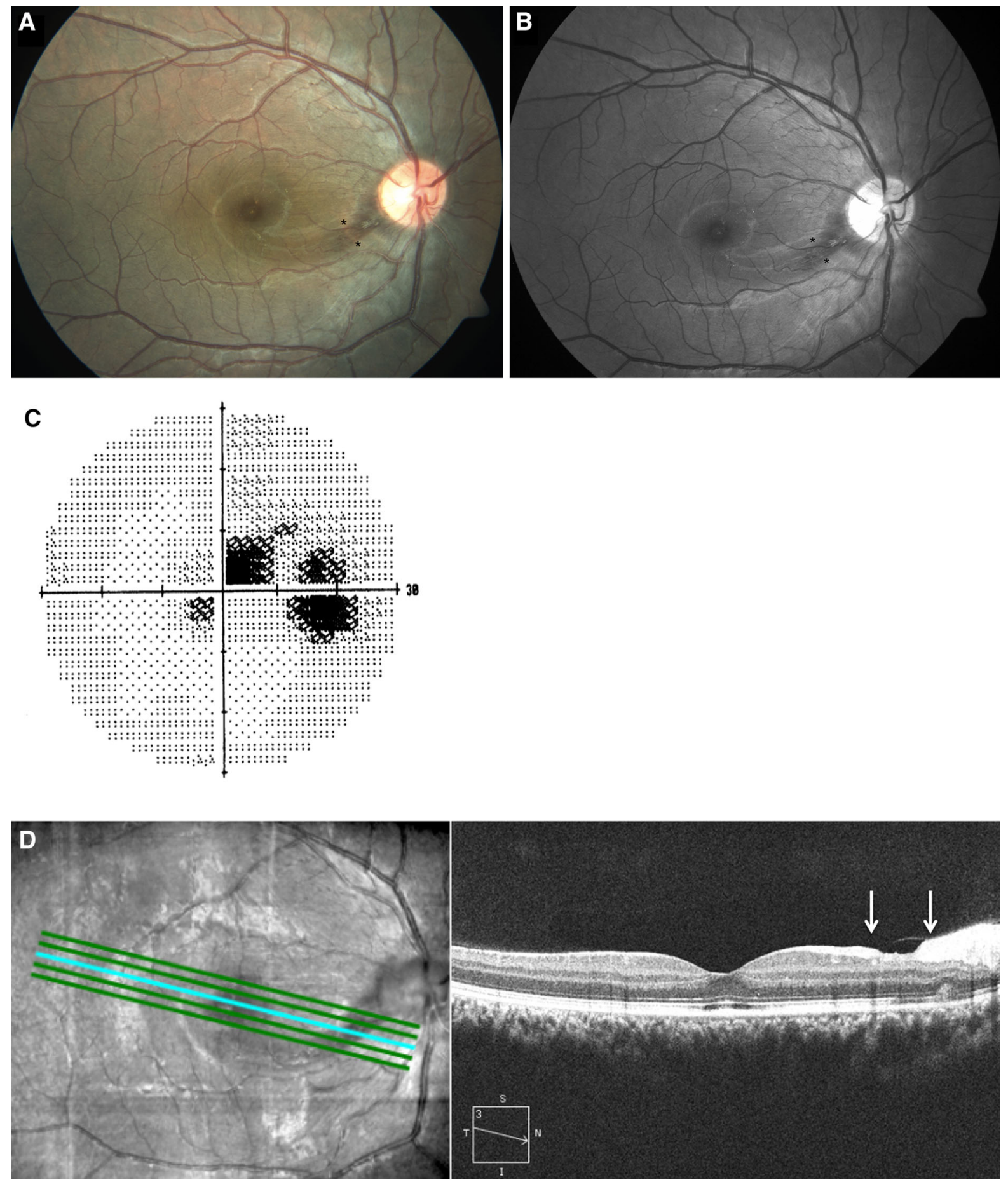

Fig. 13 Color fundus photograph (a) and red-free image (b) of a patient with Behçet uveitis show wedge-shaped peripapillary nerve fiber layer bundle defect demarcated by the asterisks. Gray-tone printout of central visual field testing with 30-2 Swedish Interactive Thresholding Algorithm Standard program of the Humphrey Field Analyzer reveals a superior arcuate centrochecal field defect corresponding to the nerve fiber layer bundle defect (c). SD OCT image of $9 \mathrm{~mm}$ scans were obtained using Cirrus HD-OCT with high-definition 5-line scan mode. A LSO fundus image shows the location of scan line through the nerve fiber bundle defect. OCT B-scan image reveals deficient nerve fiber layer in the area demarcated by the white arrows $(\mathbf{d})$ 
Table 5 Key OCT features of ocular toxoplasmosis

\section{Active retinochoroidal lesion}

Increased retinal reflectivity and thickening of the lesion site [165, 170, 171]

RPE - choriocapillaris/choroidal optical shadowing [165, 170, 171]

Thickening of the posterior hyaloid, partial or complete posterior vitreous detachment over the retinochoroidal lesion [165, 170, 171]

Multiple, small, granular hyperreflective spots on the vitreoretinal interface in the vicinity of the retinochoroidal lesion [166, 168]

Retinochoroidal scar

Decreased retinal thickness $[165,171]$

Focal choriocapillaris/choroidal relative hyperreflectivity [165, 171]

Complete posterior vitreous detachment $[165,171]$

\section{Punctate outer retinal toxoplasmosis}

Hyperreflective inner retina and thinning of the lesion site [166]

RPE - choriocapillaris/choroidal optical shadowing [166]

Thickening of the posterior hyaloid and focal detachment of the posterior hyaloid over the lesion site [166]

\section{Congenital toxoplasmosis}

Retinal thinning [169]

RPE hyperreflectivity [169]

Excavation [169]

Intraretinal cysts [169]

IS/OS inner segment/outer segment, RPE retinal pigment epithelium, ILM inner limiting membrane, IPL inner plexiform layer, $B M$ bruch membrane, ELM external limiting membrane, $O N L$ outer nuclear layer, $F A F$ fundus autofluorescence, $F A$ fluorescein angiography, ICGA indocyanine green angiography

and is characterized by small gray-white lesions that develop at the level of the outer retinal layers and the RPE. Figure 14 shows a color fundus photograph and TD OCT scan in a patient with PORT. In one study, OCT 3 scans showed thickening and focal detachment of the posterior hyaloid over PORT lesions (Fig. 14). The posterior hyaloid contained irregular hyperreflective formations in most patients. At the lesion site inner retinal layers were abnormally hyperreflective and some degree of RPE-choriocapillaris/choroidal optical shadowing was observed (Fig. 14). The retina was thinned at the lesion site in most patients. In some patients tractional maculopathy was evident due to incomplete vitreofoveal separation. During the follow-up expanded separation of the posterior hyaloid, epiretinal hyperreflective formation, inner retinal surface increased reflectivity, and disorganized retinal signaling at the lesion site associated with subjacent choriocapillaris/choroidal relative hyperreflectivity were documented [166]. In another study, OCT findings of macular PORT lesions were defined. A consistent finding on TD OCT line scan through the active lesion was attachment of the RPE- choriocapillaris layer and the neurosensory retina over the lesion with inflammatory retinal infiltrate in the deeper retinal layers and minimal intraretinal fluid in the macular area. Other findings were thickening and elevation of the RPE, focal hyperreflectivity limited to beneath the RPE, and associated minimal subretinal fluid in the macular area [167].

An additional finding detected by OCT in one patient with ocular toxoplasmosis is the appearance of multiple, small, granular, gray deposits on the vitreoretinal interface in the vicinity of acute retinochoroiditis focus [168]. The case reported by Guagnini et al. [168] showed that such deposits may appear away from the active toxoplasmic focus along retinal vessels and the macula. Deposits disappeared with combined, systemic, antiparasitic and corticosteroid treatments. OCT was helpful in following the resolution of these lesions.

Structural changes in the macula in congenital toxoplasmosis have been described using TD OCT and consisted of retinal thinning, RPE hyperreflectivity, excavation, intraretinal cysts, and fibrosis. Patients with better visual acuity than expected were shown to 
have parafoveal involvement or an intact neurosensory layer [169].

It has also been shown that OCT imaging can distinguish between active and scarred toxoplasmic lesions. The main features of active toxoplasmosis lesions were summarized as a highly reflective intraretinal area corresponding to the area of retinitis, thickening and detachment of the posterior hyaloid over the lesion, irregular hyperreflective formation, and shadowing of the underlying choroidal tissue [170].

Diniz et al. [171] defined SD OCT features of the retinochoroidal lesion, macula and vitreous and compared them with TD OCT scans in patients with active ocular toxoplasmosis. Ten eyes of ten patients were
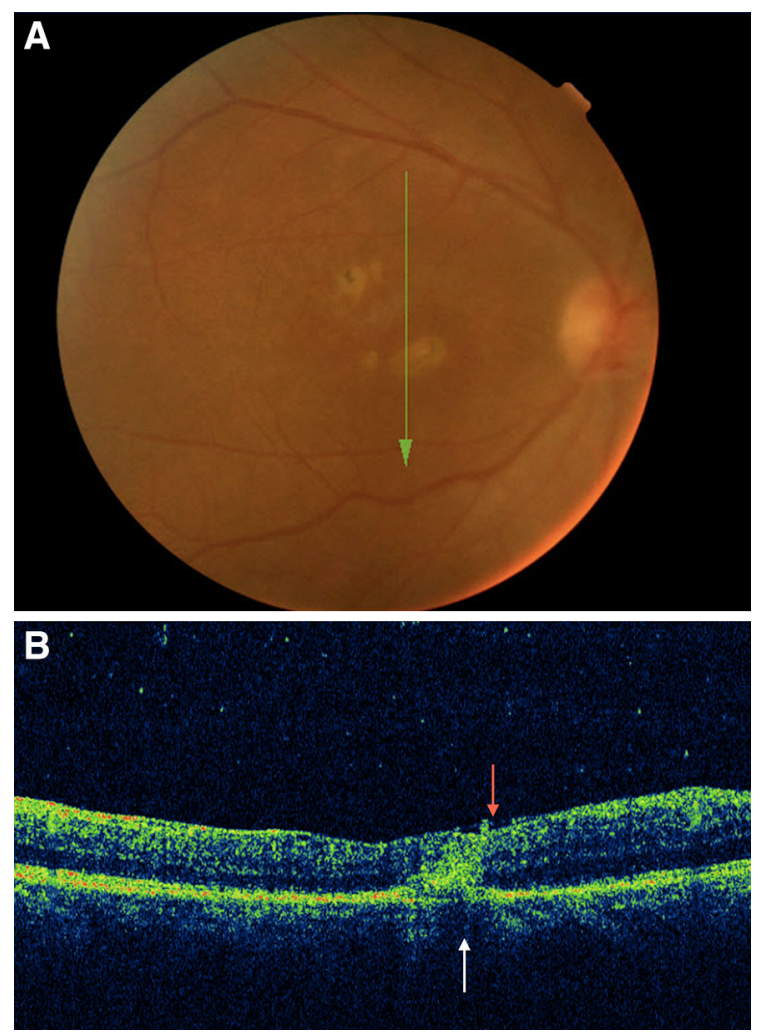

Fig. 14 Color fundus photograph shows small gray-white lesions at the level of the outer retinal layers and the RPE in an eye with punctate outer retinal toxoplasmosis (PORT); green arrow indicates the location and direction of the OCT scan line (a). TD OCT scan demonstrates abnormally hyperreflective inner retinal layers at the lesion site, the red arrow indicates thickening and focal detachment of the posterior hyaloid over the PORT lesion, and the white arrow denotes RPE-choriocapillaris/choroidal optical shadowing at the lesion site (b) analyzed. Common to all active retinochoroidal lesions was the presence of increased retinal reflectivity and thickness of the lesion site (Fig. 15). Some patients showed sublesional subretinal fluid accumulation. Both RPE and choriocapillaris shadowing, occurring as a consequence of increased hyperreflectivity of the inner retinal layers was observed. At 6-week follow-up, there was a significant decrease of the retinal thickness at the lesion site. All patients remained with a disorganization of retinal layers due to scar formation. There was IS/OS line interruption temporal to the fovea and ERM formation near the retinochoroiditis lesion in some patients. Macular serous detachments were identified in half of the patients. The presence of fluid at baseline had an indirect correlation to the distance of the retinochoroidal lesion to the fovea. Additional findings involving the macula were RPE detachment beneath the subretinal fluid and vitreomacular traction. Partial or complete posterior vitreous detachment was evident in some patients at baseline and ended with progressive separation of the hyaloid at 6-weeks (Fig. 15). Vitreous detachment occurred in all patients except one at the end of the follow-up. SD OCT had higher detection rates of macular serous retinal detachments, perilesional fluid, IS/OS junction line damage, and ERMs, and was superior in the identification of the posterior hyaloid and in assessing the vitreous status when compared to TD OCT. Figure 15 shows a highdefinition SD OCT scan of a patient with recurrence of toxoplasmic retinochoroiditis.

\section{Miscellaneous}

Morphological characteristics and their importance in the diagnosis and management of posterior uveitis entities have been emphasized by Gallagher et al. [172]. There are also reports on the evaluation of isolated posterior segment findings in patients with posterior uveitis. An OCT scan through choroidal granulomas caused by tuberculosis revealed a distinctive feature of attachment between the retinal RPEchoriocapillaris layer and the neurosensory retina over the granuloma and was described as a 'contact' sign. This was associated with surrounding subretinal fluid and inflammatory infiltrate in the deeper retinal layers [173].

In one study, Mahendradas et al. [174] reported on the documentation of a choroidal granuloma using the 
EDI SD OCT scans in an eye clinically diagnosed with retinochoroiditis. The authors emphasized the advantage of choroidal imaging using the EDI technique in facilitating the diagnosis of a choroidal mass lesion not visible clinically.

Optical coherence tomography findings of residual damage from retinal cotton wool spots that develop in noninfectious retinopathy in patients infected with human immunodeficiency virus were also described [175-177]. Resolution of cotton wool spots was found to be associated with permanent retinal destruction as shown by simultaneous SD OCT and SLO images. The greatest loss of thickness was seen in the retinal ganglion cell layer (GCL) with a $43 \%$ reduction in thickness. There was a statistically significant thinning of the RNFL, GCL, inner plexiform, INL, and outer plexiform layers. Another finding was the displacement of the ONL toward the retinal surface resulting in an apparent increase in thickness of the ONL [177].

Mahendradas et al. [178] used high-definition SD OCT (Spectralis OCT) to study intraocular cysticercosis. SD OCT was used to document an intravitreal and a subretinal cyst in one patient each. The intravitreal cyst was lying freely in the vitreous and the cyst wall appeared as a highly reflective coat surrounding a space of hyporeflectivity in which there was a hyperreflective spot. The hyperreflective spot was suggestive of the scolex/larva. High-definition SD OCT of the subretinal cyst showed a hyperreflective coat with an intensively hyperreflective portion in it. The hyperreflective portion was again suggestive of a scolex/larva. The posterior extent of the subretinal cyst could not be documented on OCT owing to the large size of the cyst.

Syphilitic posterior placoid choroidopathy has also been studied by OCT examination. Meira-Freitas et al. [179] reported on a patient with a central subretinal placoid yellowish lesion in one eye. A hypofluorescent spot corresponding to the lesion and confluent small dots extending to the periphery, without any correspondence on ophthalmoscopy were revealed by ICGA. Dots with similar distribution were also documented by ICGA in the other eye even though no lesion was observed clinically. An increased reflectance and thickening of the RPE-choriocapillaris complex corresponding to the lesion was documented by TD OCT. OCT returned to normal along with improvement of the lesion and visual acuity after treatment with intravenous antibiotic. Other OCT findings reported in eyes with syphilitic posterior placoid choroidopathy include increased thickening of the neuroretina and underlying layers in one patient and subretinal fluid at the macula in another patient, with restoration of the normal chorioretinal architecture in both after treatment [180]. Eandi et al. [181] recently reported on 16 new cases with acute syphilitic posterior placoid chorioretinitis and reviewed the literature on this disease. OCT was performed with the Stratus OCT and scans through the area of the lesion acquired in eight patients failed to show intraretinal, subphotoreceptor, or sub-RPE fluid. Despite their findings Eandi et al. disclosed an overall prevalence of subretinal fluid in 11 of 93 eyes (11.8\%) reported in the literature. Furthermore, a recent study Pichi et al. [182] focused on 30 eyes of 19 confirmed cases with acute syphilitic posterior placoid chorioretinopathy from 12 separate international centers. SD OCT was performed at different stages of the disease and showed uniform presence of a small amount of fluid under the fovea at 1-2 days after presentation in 13 of the 30 eyes. The subretinal fluid was transient, resolving quickly before the treatment in each of the eyes. At about one week after presentation, SD OCT delineated an irregular hyperreflectivity with nodular elevations at the junction of the photoreceptors and the RPE in all eyes. This was associated with segmental loss of the IS/OS band but no evidence of new or persistent fluid under the fovea at this stage. The ELM remained intact in 29 of 30 eyes. SD OCT scans taken at one month after therapy showed complete resolution of the pathologic findings of the macula in 28 of the 30 eyes, with restoration of the IS/OS junction, the RPE, and the ELM, and disappearance of the hyperreflective dots in the choroid. Only two of the 30 eyes had a vision at the last follow-up of 20/200 which, in each case, was associated with persistent disruption of $\geq 1$ of the outer retinal layers as visualized on SD OCT.

Aggio et al. [183] reported on TD OCT and mfERG findings in a patient with unilateral acute idiopathic maculopathy. In the acute phase, OCT showed abnormal heterogenous hyperreflectivity and thickening at the level of the outer retina and RPE in the fovea. Two months after presentation, OCT demonstrated a subfoveal area of homogenous hyperreflectivity that extended from the RPE to the outer retinal layers with posterior shadowing and points of hyperreflectivity at several different layers of the neurosensory retina, including superficial layers. Foveal retinal thickness 

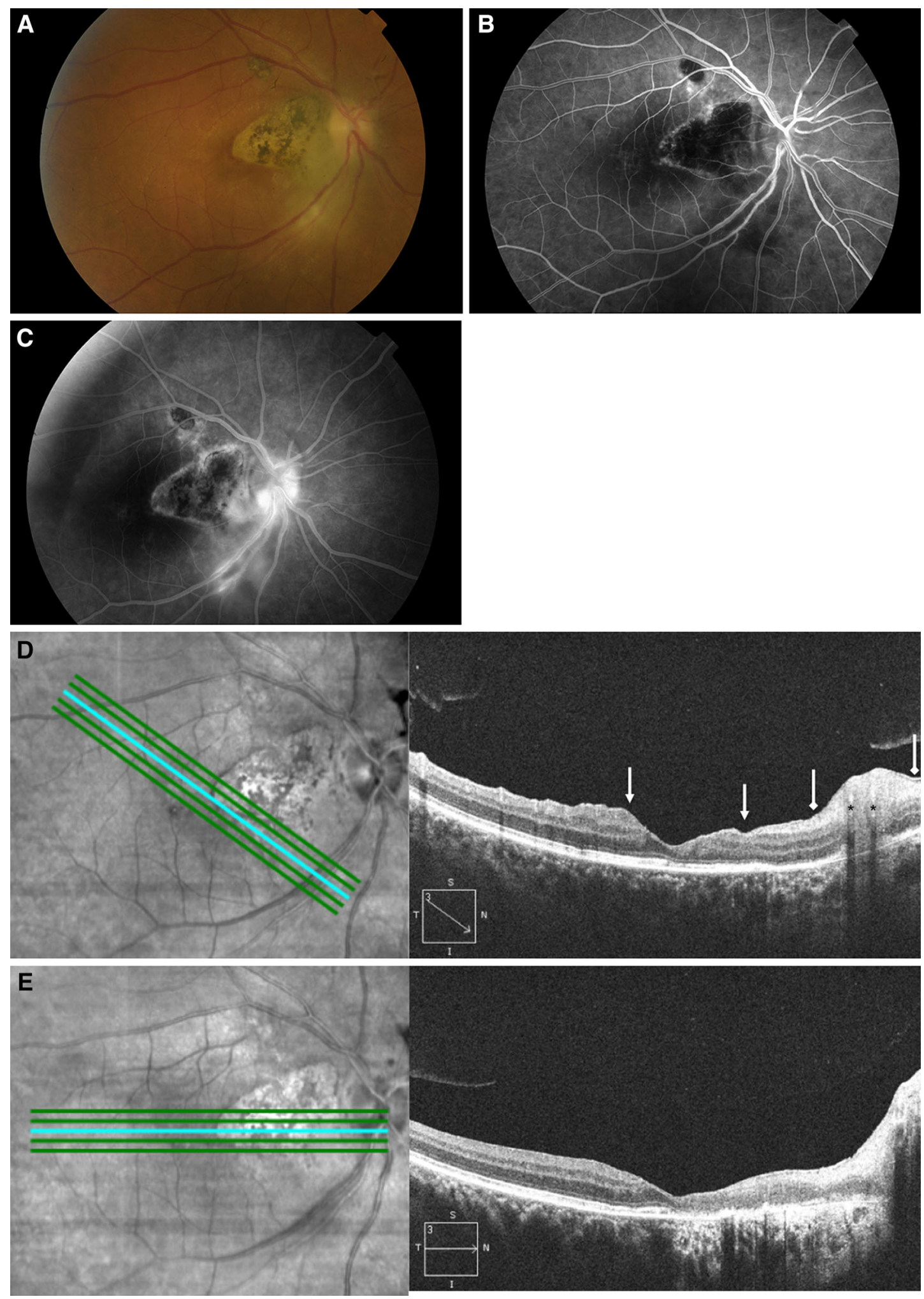
4Fig. 15 Color fundus photograph of the right eye shows active toxoplasmic retinochoroiditis along the inferior temporal vascular arcade in an eye with a pre-existing retinochoroidal scars (a). Arteriovenous phase fluorescein angiogram shows hypofluorescence of the active lesion and the scars with hyperfluorescent borders (b). Late-phase fluorescein angiogram shows hyperfluorescence of the active lesion, focal staining of the inferotemporal retinal artery and vein walls, focal disc staining, and irregular hyperfluorescence of the scars (c). SD OCT image of $9 \mathrm{~mm}$ scans were obtained using Cirrus HD-OCT with high-definition 5-line scan mode. LSO fundus image shows the location of scan line through the retinochoroiditis focus. OCT B-scan image reveals thickening of the retina and hyperreflectance of inner retinal layers and RPE-choriocapillaris/choroidal optical shadowing at the active lesion site demarcated by the diamond arrows. There is thickening and detachment of the posterior hyaloid over the lesion. Asterisks indicate shadowing of the retinal artery and vein. The same scan line passes along the border of the pigmented retinochoroidal scar and the fovea (arrows) (d). LSO fundus image shows the location of scan line through the retinochoroidal scar. OCT B-scan image reveals a disorganized and thinned retina with focal choriocapillaris/choroidal relative hyperreflectivity. Complete posterior vitreous detachment is evident across the scan (e)

decreased with resolution. Abnormal mfERG readings also returned to normal with disease resolution.

\section{Conclusion}

With the advent of OCT technology and the change from TD to SD signal detection, noninvasive in vivo imaging of the retina in detail has become possible. This technology has revolutionized the qualitative and quantitative assessment of posterior segment pathologies associated with uveitis, especially inflammatory macular edema. As it allows fast noninvasive, noncontact, real-time, and reproducible imaging of macular anatomy, OCT has now become a standard ancillary test for detecting and monitoring uveitic macular edema. Physiopathology of uveitic maculopathies has been better understood since the introduction of OCT, and classification and prognostication have become possible. Determination of an intact IS/ OS line has been proven to be a good predictor of visual recovery and prognosis in uveitic macular edema. Application of OCT technology to uveitis practice has also shed light on the physiopathology of several uveitis entities. High-resolution OCT imaging of morphological changes in the retina, vitreoretinal interface, and choroid have led to a better understanding of disease processes and distinctive features of these entities. With the introduction of the EDI OCT technique, visualization of the choroid and choriocapillaries has also become possible, providing information not available with other imaging methods, on the primary focus of inflammation in some uveitic diseases. Thus, OCT imaging has become an indispensable tool for both the differential diagnosis and follow-up of inflammatory diseases of the retina and choroid. Future advances in this field will enable visualization of the retinal and choroidal vessels in detail and high-penetration OCT systems will allow high-contrast imaging of the choroid.

\section{References}

1. Huang D, Swanson EA, Lin CP et al (1991) Optical coherence tomography. Science 254:1178-1181

2. Hee MR, Izatt JA, Swanson EA et al (1995) Optical coherence tomography of the human retina. Arch Ophthalmol 113:325-332

3. Hee MR, Puliafito CA, Wong C et al (1995) Quantitative assessment of macular edema with optical coherence tomography. Arch Ophthalmol 113:1019-1029

4. Hee HR, Puliafito CA, Wong C et al (1995) Optical coherence tomography of macular holes. Ophthalmology 102:748-756

5. Hee MR, Puliafito CA, Wong C et al (1995) Optical coherence tomography of central serous chorioretinopathy. Am J Ophthalmol 120:65-74

6. Hee MR, Baumal CR, Puliafito CA et al (1996) Optical coherence tomography of age-related macular degeneration and choroidal neovascularization. Ophthalmology 103:1260-1270

7. Yi K, Chen TC, de Boer JF (2006) Spectral domain optical coherence tomography. Tech Ophthalmol 4:170-174

8. Fercher AF, Hitzenberger CK, Kamp G, Elzaiat SY (1995) Measurement of intraocular distances by backscattering spectral interferometry. Opt Commun 117:43-48

9. Wojtkowski M, Leitgeb R, Kowalczyk A et al (2002) In vivo human retinal imaging by Fourier domain optical coherence tomography. J Biomed Opt 7:457-463

10. Drexler W, Sattmann H, Hermann B et al (2003) Enhanced visualization of macular pathology with the use of ultrahigh resolution optical coherence tomography. Arch Ophthalmol 121:695-706

11. Nassif N, Cense B, Park BH et al (2004) In vivo human retinal imaging by ultrahigh-speed spectral domain optical coherence tomography. Opt Lett 29:480-482

12. Chen TC, Cense B, Pierce MC et al (2005) Spectral domain optical coherence tomography: ultra-high speed, ultra-high resolution ophthalmic imaging. Arch Ophthalmol 123:1715-1720

13. Cense B, Nassif N, Chen T et al (2004) Ultrahigh-resolution high-speed retinal imaging using spectral-domain optical coherence tomography. Opt Express 12:2435-2447

14. Wojtkowski M, Srinivasan V, Ko T et al (2004) Ultrahighresolution, high-speed, Fourier domain optical coherence 
tomography and methods for dispersion compensation. Opt Express 12:2404-2422

15. Hangai M, Yamamoto M, Sakamoto A, Yoshimura N (2009) Ultrahigh-resolution versus speckle noise-reduction in spectral-domain optical coherence tomography. Opt Express 17:4221-4235

16. Kiernan DF, Mieler WF, Hariprasad SM (2010) Spectraldomain optical coherence tomography: a comparison of modern high-resolution retinal imaging systems. Am J Ophthalmol 149:18-31

17. Vienola KV, Braaf B, Sheehy CK et al (2012) Real-time eye motion compensation for OCT imaging with tracking SLO. Biomed Opt Express 3:2950-2963

18. Giani A, Pellegrini M, Invernizzi A et al (2012) Aligning scan locations from consecutive spectral-domain optical coherence tomography examinations: a comparison among different strategies. Invest Ophthalmol Vis Sci 53:7637-7643

19. Srinivasan VJ, Monson BK, Wojtkowski M et al (2008) Characterization of outer retinal morphology with highspeed, ultrahigh-resolution optical coherence tomography. Invest Ophthalmol Vis Sci 49:1571-1579

20. Spaide RF, Curcio CA (2011) Anatomical correlates to the bands seen in the outer retina by optical coherence tomography: literature review and model. Retina 31: 1609-1619

21. Potsaid B, Gorczynska I, Srinivasan VJ et al (2008) Ultrahigh speed spectral/Fourier domain OCT ophthalmic imaging at 70,000 to 312,500 axial scans per second. Opt Express 16:15149-15169

22. Götzinger E, Pircher M, Baumann B et al (2008) Retinal nerve fiber layer birefringence evaluated with polarization sensitive spectral domain OCT and scanning laser polarimetry: a comparison. J Biophotonics 1:129-139

23. Cense B, Chen TC, Nassif N et al (2006) Ultra-high speed and ultra-high resolution spectral-domain optical coherence tomography and optical Doppler tomography in ophthalmology. Bull Soc Belge Ophtalmol 302:123132

24. van Velthoven ME, Faber DJ, Verbraak FD et al (2007) Recent developments in optical coherence tomography for imaging the retina. Prog Retin Eye Res 26:57-77

25. Margolis R, Spaide RF (2009) A pilot study of enhanced depth imaging optical coherence tomography of the choroid in normal eyes. Am J Ophthalmol 147:811-815

26. Spaide RF, Koizumi H, Pozonni MC (2008) Enhanced depth imaging spectral-domain optical coherence tomography. Am J Ophthalmol 146:496-500

27. Podoleanu AG, Dobre GM, Webb DJ, Jackson DA (1997) Simultaneous en-face imaging of two layers in the human retina by low coherence reflectometry. Opt Lett 22:10391041

28. Podoleanu AG, Seeger M, Dobre GM et al (1998) Transversal and longitudinal images from the retina of the living eye using low coherence reflectometry. J Biomed Opt 3:12-20

29. Podoleanu AG, Dobre GM, Seeger M et al (1998) Low coherence interferometry for en-face imaging of the retina. Lasers and Light 8:187-192

30. Podoleanu AG, Jackson DA (2002) Combined optical coherence tomography and scanning laser ophthalmoscopy. Electron Lett 34:1088-1090
31. van Velthoven MEJ, Verbraak FD, Yannuzzi LA et al (2006) Imaging the retina by en-face optical coherence tomography. Retina 26:129-136

32. Michaely R, Bachmann AH, Villiger ML et al (2007) Vectorial reconstruction of retinal blood flow in three dimensions measured with high resolution resonant Doppler Fourier domain optical coherence tomography. J Biomed Opt 12:041213

33. Yasuno Y, Hong Y, Makita S, Yamanari M, Akiba M, Miura M, Yatagai T (2007) In vivo high-contrast imaging of deep posterior eye by 1-microm swept source optical coherence tomography and scattering optical coherence angiography. Opt Express 15:6121-6139

34. Hong Y, Makita S, Yamanari M et al (2007) Threedimensional visualization of choroidal vessels by using standard and ultra-high resolution scattering optical coherence angiography. Opt Express 15:7538-7550

35. Unterhuber A, Povazay B, Hermann B et al (2005) In vivo retinal optical coherence tomography at $1040 \mathrm{~nm}-$ enhanced penetration into the choroid. Opt Express 13: 3252-3258

36. Lee EC, de Boer JF, Mujat M et al (2006) In vivo optical frequency domain imaging of human retina and choroid. Opt Express 14:4403-4411

37. Povazay B, Hermann B, Unterhuber A et al (2007) Threedimensional optical coherence tomography at $1050 \mathrm{~nm}$ versus $800 \mathrm{~nm}$ in retinal pathologies: enhanced performance and choroidal penetration in cataract patients. J Biomed Opt 12:041211

38. Gupta V, Gupta P, Singh R et al (2008) Spectral-domain Cirrus high-definition optical coherence tomography is better than time-domain Stratus optical coherence tomography for evaluation of macular pathologic features in uveitis. Am J Ophthalmol 145:1018-1022

39. Agarwal A, Ashokkumar D, Jacob S et al (2009) Highspeed optical coherence tomography for imaging anterior chamber inflammatory reaction in uveitis: clinical correlation and grading. Am J Ophthalmol 147:413-416

40. Hashida N, Terubayashi A, Ohguro N (2011) Anterior segment optical coherence tomography findings of presumed intraocular tuberculosis. Cutan Ocul Toxicol 30:75-77

41. Roesel M, Henschel A, Heinz C et al (2008) Time-domain and spectral-domain optical coherence tomography in uveitic macular edema. Am J Ophthalmol 146:626-627

42. Iannetti L, Pesci FR, Spinucci G et al (2012) Reproducibility of retinal thickness measurements in eyes with uveitic macular oedema using Spectralis optical coherence tomography. Acta Ophthalmol 90:e490-e491

43. Antcliff RJ, Stanford MR, Chauhan DS et al (2000) Comparison between optical coherence tomography and fundus fluorescein angiography for the detection of cystoid macular edema in patients with uveitis. Ophthalmology 107:593-599

44. Hassenstein A, Bialasiewicz AA, Richard G (2000) Optical coherence tomography in uveitis patients. Am J Ophthalmol 130:669-670

45. Markomichelakis NN, Halkiadakis I, Pantelia E et al (2004) Patterns of macular edema in patients with uveitis: qualitative and quantitative assessment using optical coherence tomography. Ophthalmology 111:946-953 
46. Byeon SH, Chu YK, Hong YT et al (2012) New insights into the pathoanatomy of diabetic macular edema: angiographic patterns and optical coherence tomography. Retina 32:1087-1099

47. Estafanous MF, Lowder CY, Kaiser PK (2005) Patterns of macular edema in uveitis patients. Ophthalmology 112:360

48. Sivaprasad S, Ikeji F, Xing W, Lightman S (2007) Tomographic assessment of therapeutic response to uveitic macular oedema. Clin Experiment Ophthalmol 35:719723

49. Iannetti L, Accorinti M, Liverani M et al (2008) Optical coherence tomography for classification and clinical evaluation of macular edema in patients with uveitis. Ocul Immunol Inflamm 16:155-160

50. Tran TH, de Smet MD, Bodaghi B et al (2008) Uveitic macular oedema: correlation between optical coherence tomography patterns with visual acuity and fluorescein angiography. Br J Ophthalmol 92:922-927

51. Al-Mezaine HS, Al-Muammar A, Kangave D, Abu ElAsrar AM (2008) Clinical and optical coherence tomographic findings and outcome of treatment in patients with presumed tuberculous uveitis. Int Ophthalmol 28:413-423

52. Iannetti L, Spinucci G, Abbouda A et al (2012) Spectraldomain optical coherence tomography in uveitic macular edema: morphological features and prognostic factors. Ophthalmologica 228:13-18

53. Ossewaarde-van Norel J, Berg EM, Sijssens KM, Rothova A (2011) Subfoveal serous retinal detachment in patients with uveitic macular edema. Arch Ophthalmol 129:158162

54. Lehpamer B, Moshier E, Goldberg N et al (2013) Subretinal fluid in uveitic macular edema: effect on vision and response to therapy. Am J Ophthalmol 155:143-149

55. Castellano CG, Stinnett SS, Mettu PS et al (2009) Retinal thickening in iridocyclitis. Am J Ophthalmol 148:341-349

56. Traill A, Stawell R, Hall A, Zamir E (2007) Macular thickening in acute anterior uveitis. Ophthalmology 114:402

57. de Lahitte GD, Terrada C, Tran TH et al (2008) Maculopathy in uveitis of juvenile idiopathic arthritis: an optical coherence tomography study. Br J Ophthalmol 92:64-69

58. Moreno-Arrones JP, Gorroño-Echebarría MB, TeusGuezala MA (2010) Macular thickening in acute anterior uveitis with a 6-month remission period. Can J Ophthalmol 45:91-92

59. Skarmoutsos F, Sandhu SS, Voros GM, Shafiq A (2006) The use of optical coherence tomography in the management of cystoid macular edema in pediatric uveitis. J AAPOS 10:173-174

60. Paroli MP, Spinucci G, Fabiani C, Pivetti-Pezzi P (2010) Retinal complications of juvenile idiopathic arthritisrelated uveitis: a microperimetry and optical coherence tomography study. Ocul Immunol Inflamm 18:54-59

61. Kump LI, Cervantes-Castañeda RA, Androudi SN, Foster CS (2005) Analysis of pediatric uveitis cases at a tertiary referral center. Ophthalmology 112:1287-1292

62. Sabri K, Saurenmann RK, Silverman ED, Levin AV (2008) Course, complications, and outcome of juvenile arthritis-related uveitis. J AAPOS 12:539-545

63. Saurenmann RK, Levin AV, Feldman BM et al (2007) Prevalence, risk factors, and outcome of uveitis in juvenile idiopathic arthritis: a long-term followup study. Arthritis Rheum 56:647-657

64. Woreta F, Thorne JE, Jabs DA et al (2007) Risk factors for ocular complications and poor visual acuity at presentation among patients with uveitis associated with juvenile idiopathic arthritis. Am J Ophthalmol 143:647-655

65. Akduman L (2009) Can we be more objective in determining the response to treatment in uveitis patients aside from recording anterior chamber reaction? Ocul Immunol Inflamm 17:265-266

66. Roesel M, Henschel A, Heinz C et al (2009) Fundus autofluorescence and spectral domain optical coherence tomography in uveitic macular edema. Graefes Arch Clin Exp Ophthalmol 247:1685-1689

67. Roesel M, Heimes B, Heinz C et al (2011) Comparison of retinal thickness and fundus-related microperimetry with visual acuity in uveitic macular oedema. Acta Ophthalmol 89:533-537

68. Georgiadou E, Moschos MM, Margetis I et al (2012) Structural and functional outcomes after treatment of uveitic macular oedema: an optical coherence tomography and multifocal electroretinogram study. Clin Exp Optom 95:89-93

69. Antcliff RJ, Spalton DJ, Stanford MR et al (2001) Intravitreal triamcinolone for uveitic cystoid macular edema: an optical coherence tomography study. Ophthalmology 108:765-772

70. Angunawela RI, Heatley CJ, Williamson TH et al (2005) Intravitreal triamcinalone acetonide for refractory uveitic cystoid macular oedema: longterm management and outcome. Acta Ophthalmol Scand 83:595-599

71. Venkatesh P, Abhas Z, Garg S, Vohra R (2007) Prospective optical coherence tomographic evaluation of the efficacy of oral and posterior subtenon corticosteroids in patients with intermediate uveitis. Graefes Arch Clin Exp Ophthalmol 245:59-67

72. Cordero Coma M, Sobrin L, Onal S et al (2007) Intravitreal bevacizumab for treatment of uveitic macular edema. Ophthalmology 114:1574-1579

73. Markomichelakis NN, Halkiadakis I, Pantelia E et al (2007) Course of macular edema in uveitis under medical treatment. Ocul Immunol Inflamm 15:71-79

74. Mackensen F, Heinz C, Becker MD, Heiligenhaus A (2008) Intravitreal bevacizumab (avastin) as a treatment for refractory macular edema in patients with uveitis: a pilot study. Retina 28:41-45

75. Lasave AF, Zeballos DG, El-Haig WM et al (2009) Shortterm results of a single intravitreal bevacizumab (avastin) injection versus a single intravitreal triamcinolone acetonide (kenacort) injection for the management of refractory noninfectious uveitic cystoid macular edema. Ocul Immunol Inflamm 17:423-430

76. Hariprasad SM, Akduman L, Clever JA et al (2009) Treatment of cystoid macular edema with the new-generation NSAID nepafenac $0.1 \%$. Clin Ophthalmol 3:147-154

77. Cervantes-Castañeda RA, Giuliari GP, Gallagher MJ et al (2009) Intravitreal bevacizumab in refractory uveitic macular edema: one-year follow-up. Eur J Ophthalmol 19:622-629

78. Deuter CM, Kötter I, Günaydin I et al (2009) Efficacy and tolerability of interferon alpha treatment in patients with 
chronic cystoid macular oedema due to non-infectious uveitis. Br J Ophthalmol 93:906-913

79. Schulze-Döbold C, Weber M (2009) Loss of visual function after repeated intravitreal injections of triamcinolone acetonide in refractory uveitic macular oedema. Int Ophthalmol 29:427-429

80. Bélair ML, Kim SJ, Thorne JE et al (2009) Incidence of cystoid macular edema after cataract surgery in patients with and without uveitis using optical coherence tomography. Am J Ophthalmol 148:128-135

81. Androudi S, Tsironi E, Kalogeropoulos C et al (2010) Intravitreal adalimumab for refractory uveitis-related macular edema. Ophthalmology 117:1612-1616

82. Soheilian M, Rabbanikhah Z, Ramezani A et al (2010) Intravitreal bevacizumab versus triamcinolone acetonide for refractory uveitic cystoid macular edema: a randomized pilot study. J Ocul Pharmacol Ther 26:199-206

83. Acharya NR, Sittivarakul W, Qian Y et al (2011) Bilateral effect of unilateral ranibizumab in patients with uveitisrelated macular edema. Retina 31:1871-1876

84. Faia LJ, Sen HN, Li Z et al (2011) Treatment of inflammatory macular edema with humanized anti-CD11a antibody therapy. Invest Ophthalmol Vis Sci 52:6919-6924

85. Bae JH, Lee CS, Lee SC (2011) Efficacy and safety of intravitreal bevacizumab compared with intravitreal and posterior sub-tenon triamcinolone acetonide for treatment of uveitic cystoid macular edema. Retina 31:111-118

86. Sugar EA, Jabs DA, Altaweel MM, Multicenter Uveitis Steroid Treatment (MUST) Trial Research Group et al (2011) Identifying a clinically meaningful threshold for change in uveitic macular edema evaluated by optical coherence tomography. Am J Ophthalmol 152:10441052

87. Payne JF, Bruce BB, Lee LB, Yeh S (2011) Logarithmic transformation of spectral-domain optical coherence tomography data in uveitis-associated macular edema. Invest Ophthalmol Vis Sci 52:8939-8943

88. Scholl S, Kirchhof J, Augustin AJ (2010) Pathophysiology of macular edema. Ophthalmologica 224(Suppl 1):8-15

89. Reichenbach A, Wurm A, Pannicke T et al (2007) Müller cells as players in retinal degeneration and edema. Graefes Arch Clin Exp Ophthalmol 245:627-636

90. Nussenblatt RB (1986) Macular alterations secondary to intraocular inflammatory disease. Ophthalmology 93:984988

91. Forooghian F, Yeh S, Faia LJ, Nussenblatt RB (2009) Uveitic foveal atrophy: clinical features and associations. Arch Ophthalmol 127:179-186

92. Maruyama Y, Kishi S (2004) Tomographic features of serous retinal detachment in Vogt-Koyanagi-Harada syndrome. Ophthalmic Surg Lasers Imaging 35:239-242

93. Parc C, Guenoun JM, Dhote R, Brezin A (2005) Optical coherence tomography in the acute and chronic phases of Vogt-Koyanagi-Harada disease. Ocul Immunol Inflamm 13:225-227

94. Tsujikawa A, Yamashiro K, Yamamoto K et al (2005) Retinal cystoid spaces in acute Vogt-Koyanagi-Harada syndrome. Am J Ophthalmol 139:670-677
95. de Smet MD, Rao NA (2005) Retinal cystoid spaces in acute Vogt-Koyanagi-Harada syndrome. Am J Ophthalmol 140:962-963

96. Yamaguchi Y, Otani T, Kishi S (2007) Tomographic features of serous retinal detachment with multilobular dye pooling in acute Vogt-Koyanagi-Harada disease. Am J Ophthalmol 144:260-265

97. Yamanaka E, Ohguro N, Yamamoto S et al (2002) Evaluation of pulse corticosteroid therapy for Vogt-KoyanagiHarada disease assessed by optical coherence tomography. Am J Ophthalmol 134:454-456

98. Lee JE, Park SW, Lee JK et al (2009) Edema of the photoreceptor layer in Vogt-Koyanagi-Harada disease observed using high-resolution optical coherence tomography. Korean J Ophthalmol 23:74-79

99. Fardeau C, Tran TH, Gharbi B et al (2007) Retinal fluorescein and indocyanine green angiography and optical coherence tomography in successive stages of Vogt-Koyanagi-Harada disease. Int Ophthalmol 27:163-172

100. Ishihara K, Hangai M, Kita M, Yoshimura N (2009) Acute Vogt-Koyanagi-Harada disease in enhanced spectraldomain optical coherence tomography. Ophthalmology 116:1799-1807

101. Ikewaki J, Kimoto K, Choshi T et al (2011) Optical coherence tomographic assessment of dynamic macular changes in patients with Vogt-Koyanagi-Harada disease. Int Ophthalmol 31:9-13

102. Gupta V, Gupta A, Gupta P, Sharma A (2009) Spectraldomain cirrus optical coherence tomography of choroidal striations seen in the acute stage of Vogt-Koyanagi-Harada disease. Am J Ophthalmol 147:148-153

103. Wu W, Wen F, Huang S et al (2007) Choroidal folds in Vogt-Koyanagi-Harada disease. Am J Ophthalmol 143: 900-901

104. Zhao C, Zhang M, Wen X et al (2009) Choroidal folds in acute Vogt-Koyanagi-Harada disease. Ocul Immunol Inflamm 17:282-288

105. Okamoto Y, Miyake Y, Horio N et al (2004) Delayed regeneration of foveal cone photopigments in Vogt-Koyanagi-Harada disease at the convalescent stage. Invest Ophthalmol Vis Sci 45:318-322

106. Vasconcelos-Santos DV, Sohn EH, Sadda S, Rao NA (2010) Retinal pigment epithelial changes in chronic Vogt-KoyanagiHarada disease: fundus autofluorescence and spectral domainoptical coherence tomography findings. Retina 30:33-41

107. Read RW, Holland GN, Rao NA et al (2001) Revised diagnostic criteria for Vogt-Koyanagi-Harada disease: report of an international committee on nomenclature. Am J Ophthalmol 131:647-652

108. Rao NA (2007) Pathology of Vogt-Koyanagi-Harada disease. Int Ophthalmol 27:81-85

109. Fong AH, Li KK, Wong D (2011) Choroidal evaluation using enhanced depth imaging spectral-domain optical coherence tomography in Vogt-Koyanagi-Harada disease. Retina 31:502-509

110. Herbort CP, Mantovani A, Bouchenaki N (2007) Indocyanine green angiography in Vogt-Koyanagi-Harada disease: angiographic signs and utility in patient follow-up. Int Ophthalmol 27:173-182 
111. Maruko I, Iida T, Sugano Y et al (2011) Subfoveal choroidal thickness after treatment of Vogt-Koyanagi-Harada disease. Retina 31:510-517

112. Yamamoto M, Nishijima K, Nakamura M, Yoshimura N (2011) Inner retinal changes in acute-phase Vogt-Koyanagi-Harada disease measured by enhanced spectral domain optical coherence tomography. Jpn J Ophthalmol 55:1-6

113. Gupta V, Gupta A, Dogra MR, Singh I (2011) Reversible retinal changes in the acute stage of sympathetic ophthalmia seen on spectral domain optical coherence tomography. Int Ophthalmol 31:105-110

114. Correnti AJ, Read RW, Kimble JA, Morris R (2010) Imaging of Dalen-Fuchs nodules in a likely case of sympathetic ophthalmia by fluorescein angiography and OCT. Ophthalmic Surg Lasers Imaging 9:1-3

115. Lofoco G, Ciucci F, Bardocci A et al (2005) Optical coherence tomography findings in a case of acute multifocal posterior placoid pigment epitheliopathy (AMPPPE). Eur J Ophthalmol 15:143-147

116. Lim LL, Watzke RC, Lauer AK, Smith JR (2006) Ocular coherence tomography in acute posterior multifocal placoid pigment epitheliopathy. Clin Experiment Ophthalmol 34:810-812

117. Scheufele TA, Witkin AJ, Schocket LS et al (2005) Photoreceptor atrophy in acute posterior multifocal placoid pigment epitheliopathy demonstrated by optical coherence tomography. Retina 25:1109-1112

118. Garg S, Jampol LM (2004) Macular serous detachment in acute posterior multifocal placoid pigment epitheliopathy. Retina 24:650-651

119. Birnbaum AD, Blair MP, Tessler HH, Goldstein DA (2010) Subretinal fluid in acute posterior multifocal placoid pigment epitheliopathy. Retina 30:810-814

120. Lee GE, Lee BW, Rao NA, Fawzi AA (2011) Spectral domain optical coherence tomography and autofluorescence in a case of acute posterior multifocal placoid pigment epitheliopathy mimicking Vogt-Koyanagi-Harada disease: case report and review of literature. Ocul Immunol Inflamm 19:42-47

121. Montero JA, Ruiz-Moreno JM, Fernandez-Munoz M (2011) Spectral domain optical coherence tomography findings in acute posterior multifocal placoid pigment epitheliopathy. Ocul Immunol Inflamm 19:48-50

122. Batioglu F, Ozmert E, Kurt R (2008) Fundus autofluorescence and spectral optical coherence tomography findings in a case with acute posterior multifocal placoid pigment epitheliopathy. Ann Ophthalmol (Skokie) 40:185-189

123. Souka AA, Hillenkamp J, Gora F et al (2006) Correlation between optical coherence tomography and autofluorescence in acute posterior multifocal placoid pigment epitheliopathy. Graefes Arch Clin Exp Ophthalmol 244:1219-1223

124. Tarabishy AB, Lowder CY (2010) Retinal pigment epithelium disturbances in acute posterior multifocal placoid pigment epitheliopathy. Eye (Lond) 24:1404-1405

125. Cheung CM, Yeo IY, Koh A (2010) Photoreceptor changes in acute and resolved acute posterior multifocal placoid pigment epitheliopathy documented by spectral-domain optical coherence tomography. Arch Ophthalmol 128:644-646

126. Amer R, Florescu T (2008) Optical coherence tomography in relentless placoid chorioretinitis. Clin Experiment Ophthalmol 36:388-390
127. Li D, Kishi S (2007) Loss of photoreceptor outer segment in acute zonal occult outer retinopathy. Arch Ophthalmol 125:1194-1200

128. Zibrandtsen N, Munch IC, Klemp K et al (2008) Photoreceptor atrophy in acute zonal occult outer retinopathy. Acta Ophthalmol 86:913-916

129. Takai Y, Ishiko S, Kagokawa H et al (2009) Morphological study of acute zonal occult outer retinopathy (AZOOR) by multiplanar optical coherence tomography. Acta Ophthalmol 87:408-418

130. Spaide RF, Koizumi H, Freund KB (2008) Photoreceptor outer segment abnormalities as a cause of blind spot enlargement in acute zonal occult outer retinopathy-complex diseases. Am J Ophthalmol 146:111-120

131. Fujiwara T, Imamura Y, Giovinazzo VJ, Spaide RF (2010) Fundus autofluorescence and optical coherence tomographic findings in acute zonal occult outer retinopathy. Retina 30:1206-1216

132. Tunoda K, Fujinami K, Miyake Y (2011) Selective abnormality of cone outer segment tip line in acute zonal occult outer retinopathy as observed by spectral-domain optical coherence tomography. Arch Ophthalmol 129:1099-1101

133. So K, Shinoda K, Matsumoto CS, Satofuka S, Imamura Y, Mizota A (2011) Focal functional and microstructural changes of photoreceptors in eyes with acute zonal occult outer retinopathy. Case Report Ophthalmol 2: 307-313

134. Ohta K, Sato A, Fukui E (2009) Spectral domain optical coherence tomographic findings at convalescent stage of acute zonal occult outer retinopathy. Clin Ophthalmol 3:423-428

135. Mkrtchyan M, Lujan BJ, Merino D et al (2012) Outer retinal structure in patients with acute zonal occult outer retinopathy. Am J Ophthalmol 153:757-768

136. Vance SK, Spaide RF, Freund KB et al (2011) Outer retinal abnormalities in acute macular neuroretinopathy. Retina 31:441-445

137. Kanis MJ, van Norren D (2006) Integrity of foveal cones in multiple evanescent white dot syndrome assessed with OCT and foveal reflection analyser. $\mathrm{Br} \mathrm{J}$ Ophthalmol 90:795-796

138. Nguyen MH, Witkin AJ, Reichel E et al (2007) Microstructural abnormalities in MEWDS demonstrated by ultrahigh resolution optical coherence tomography. Retina 27:414-418

139. Li D, Kishi S (2009) Restored photoreceptor outer segment damage in multiple evanescent white dot syndrome. Ophthalmology 116:762-770

140. Forooghian F, Stetson PF, Gross NE, Meyerle CB (2010) Quantitative assessment of photoreceptor recovery in atypical multiple evanescent white dot syndrome. Ophthalmic Surg Lasers Imaging 41:S77-S80. doi:10.3928/1542 8877-20101031-06

141. Hua R, Chen K, Liu LM et al (2012) Multi-modality imaging on multiple evanescent white dot syndrome-a Spectralis study. Int J Ophthalmol 5:644-647

142. Sikorski BL, Wojtkowski M, Kaluzny JJ et al (2008) Correlation of spectral optical coherence tomography with fluorescein and indocyanine green angiography in multiple evanescent white dot syndrome. Br J Ophthalmol 92: 1552-1557 
143. Hangai M, Fujimoto M, Yoshimura N (2009) Features and function of multiple evanescent white dot syndrome. Arch Ophthalmol 127:1307-1313

144. Aoyagi R, Hayashi T, Masai A et al (2012) Subfoveal choroidal thickness in multiple evanescent white dot syndrome. Clin Exp Optom 95:212-217

145. Amin HI (2006) Optical coherence tomography findings in multiple evanescent white dot syndrome. Retina 26:483484

146. Yasuno Y, Okamoto F, Kawana K et al (2009) Investigation of multifocal choroiditis with panuveitis by threedimensional high-penetration optical coherence tomography. J Biophotonics 2:435-441

147. Rao VG, Rao GS, Narkhede NS (2011) Flare up of choroiditis and choroidal neovasculazation associated with punctate inner choroidopathy during early pregnancy. Indian J Ophthalmol 59:145-148

148. Chan WM, Lai TY, Liu DT, Lam DS (2007) Intravitreal bevacizumab (avastin) for choroidal neovascularization secondary to central serous chorioretinopathy, secondary to punctate inner choroidopathy, or of idiopathic origin. Am J Ophthalmol 143:977-983

149. Stepien KE, Carroll J (2011) Using spectral-domain optical coherence tomography to follow outer retinal structure changes in a patient with recurrent punctate inner choroidopathy. J Ophthalmol 2011:753741

150. van Velthoven ME, Ongkosuwito JV, Verbraak FD et al (2006) Combined en-face optical coherence tomography and confocal ophthalmoscopy findings in active multifocal and serpiginous chorioretinitis. Am J Ophthalmol 141: 972-975

151. Vance SK, Khan S, Klancnik JM, Freund KB (2011) Characteristic spectral-domain optical coherence tomography findings of multifocal choroiditis. Retina 31:717723

152. Kotsolis AI, Killian FA, Ladas ID, Yannuzzi LA (2010) Fluorescein angiography and optical coherence tomography concordance for choroidal neovascularisation in multifocal choroidtis. Br J Ophthalmol 94:1506-1508

153. Zolf R, Glacet-Bernard A, Benhamou N et al (2002) Imaging analysis with optical coherence tomography: relevance for submacular surgery in high myopia and in multifocal choroiditis. Retina 22:192-201

154. Punjabi OS, Rich R, Davis JL et al (2008) Imaging serpiginous choroidopathy with spectral domain optical coherence tomography. Ophthalmic Surg Lasers Imaging 39(4Suppl):S95-S98

155. Arantes TE, Matos K, Garcia CR et al (2011) Fundus autofluorescence and spectral domain optical coherence tomography in recurrent serpiginous choroiditis: case report. Ocul Immunol Inflamm 19:39-41

156. Witkin AJ, Duker JS, Ko TH et al (2005) Ultrahigh resolution optical coherence tomography of birdshot retinochoroidopathy. Br J Ophthalmol 89:1660-1661

157. Monnet D, Levinson RD, Holland GN et al (2007) Longitudinal cohort study of patients with birdshot chorioretinopathy. III. Macular imaging at baseline. Am J Ophthalmol 144:818-828

158. Birch DG, Williams PD, Callanan D et al (2010) Macular atrophy in birdshot retinochoroidopathy: an optical coherence tomography and multifocal electroretinography analysis. Retina 30:930-937

159. Forooghian F, Gulati N, Jabs DA (2010) Restoration of retinal architecture following systemic immunosuppression in birdshot chorioretinopathy. Ocul Immunol Inflamm 18:470-471

160. Iaccarino G, Cennamo G, Forte R, Cennamo G (2009) Evaluation of posterior pole with echography and optical coherence tomography in patients with Behçet's disease. Ophthalmologica 223:250-255

161. Atmaca LS, Batioglu F, Müftüoglu O (2003) Fluorescein angiography and optical coherence tomography in ocular Behçet's disease. A preliminary study. Adv Exp Med Biol 528:355-360

162. Takeuchi M, Iwasaki T, Kezuka T et al (2010) Functional and morphological changes in the eyes of Behçet's patients with uveitis. Acta Ophthalmol 88:257-262

163. Unoki N, Nishijima K, Kita M et al (2010) Structural changes of fovea during remission of Behçet's disease as imaged by spectral domain optical coherence tomography. Eye (Lond) 24:969-975

164. Sheets CW, Grewal DS, Greenfield DS (2009) Ocular toxoplasmosis presenting with focal retinal nerve fiber atrophy simulating glaucoma. J Glaucoma 18:129-131

165. Oréfice JL, Costa RA, Oréfice F et al (2007) Vitreoretinal morphology in active ocular toxoplasmosis: a prospective study by optical coherence tomography. Br J Ophthalmol 91:773-780

166. Oréfice JL, Costa RA, Campos W et al (2006) Third-generation optical coherence tomography findings in punctate retinal toxoplasmosis. Am J Ophthalmol 142:503-505

167. de Souza EC, Casella AM (2009) Clinical and tomographic features of macular punctate outer retinal toxoplasmosis. Arch Ophthalmol 127:1390-1394

168. Guagnini AP, De Potter P, Levecq L, Kozyreff A (2007) Atypical spherical deposition on vitreoretinal interface associated with toxoplasmic chorioretinitis. Graefes Arch Clin Exp Ophthalmol 245:158-160

169. Garg S, Mets MB, Bearelly S, Mets R (2009) Imaging of congenital toxoplasmosis macular scars with optical coherence tomography. Retina 29:631-637

170. Monnet D, Averous K, Delair E, Brézin AP (2009) Optical coherence tomography in ocular toxoplasmosis. Int J Med Sci 6:137-138

171. Diniz B, Regatieri C, Andrade R, Maia A (2011) Evaluation of spectral domain and time domain optical coherence tomography findings in toxoplasmic retinochoroiditis. Clin Ophthalmol 5:645-650

172. Gallagher MJ, Yilmaz T, Cervantes-Castañeda RA, Foster CS (2007) The characteristic features of optical coherence tomography in posterior uveitis. $\mathrm{Br} \mathrm{J}$ Ophthalmol 91:1680-1685

173. Salman A, Parmar P, Rajamohan M et al (2006) Optical coherence tomography in choroidal tuberculosis. Am J Ophthalmol 142:170-172

174. Mahendradas P, Avadhani K, Yadav NK et al (2010) Role of Spectralis HRA + OCT spectral domain optical coherence tomography in the diagnosis and management of fungal choroidal granuloma. Ocul Immunol Inflamm 18:408-410 
175. Kozak I, Bartsch DU, Cheng L, Freeman WR (2006) In vivo histology of cotton-wool spots using high-resolution optical coherence tomography. Am J Ophthalmol 141:748-750

176. Kozak I, Bartsch DU, Cheng L, Freeman WR (2007) Hyperreflective sign in resolved cotton wool spots using high-resolution optical coherence tomography and optical coherence tomography ophthalmoscopy. Ophthalmology 114:537-543

177. Gomez ML, Mojana F, Bartsch DU, Freeman WR (2009) Imaging of long-term retinal damage after resolved cotton wool spots. Ophthalmology 116:2407-2414

178. Mahendradas P, Avadhani K, Yadav NK et al (2011) Highdefinition spectral-domain optical coherence tomography of intravitreal and subretinal cysticercus cysts in intraocular cysticercosis. Retina 31:2132-2133

179. Meira-Freitas D, Farah ME, Höfling-Lima AL, Aggio FB (2009) Optical coherence tomography and indocyanine green angiography findings in acute syphilitic posterior placoid choroidopathy: case report. Arq Bras Oftalmol 72:832-835

180. Joseph A, Rogers S, Browning A et al (2007) Syphilitic acute posterior placoid chorioretinitis in nonimmunocompromised patients. Eye (Lond) 21:1114-1119

181. Eandi CM, Neri P, Adelman RA et al (2012) On behalf of International syphilis study group. Acute syphilitic posterior placoid chorioretinitis: report of a case series and comprehensive review of the literature. Retina 32:19151941

182. Pichi F, Ciardella AP, Cunningham ET, et al (2013). Spectral domain optical coherence tomography findings in patients with acute syphilitic posterior placoid chorioretinopathy. Retina (in press)

183. Aggio FB, Farah ME, Meirelles RL, de Souza EC (2006) STRATUS OCT and multifocal ERG in unilateral acute idiopathic maculopathy. Graefes Arch Clin Exp Ophthalmol 244:510-516 